Groups Geom. Dyn. 8 (2014), 69-95

DOI $10.4171 / \mathrm{GGD} / 217$
Groups, Geometry, and Dynamics

(C) European Mathematical Society

\title{
Geometric two-dimensional duality groups
}

\author{
Risto Atanasov
}

\begin{abstract}
We consider a finite, aspherical, 2-dimensional Cohen-Macaulay simplicial complex $\Delta$ and we find additional conditions that imply the universal cover $\widetilde{\Delta}$ has one end. In order to find these additional conditions we use a form of "Zeeman Duality". The context is an attempt to better understand duality groups.
\end{abstract}

Mathematics Subject Classification (2010). 20F65.

Keywords. Cohen-Macaulay complex, duality group.

\section{Introduction}

1.1. The motivating question. Let $\Delta$ be a connected finite $n$-dimensional simplicial complex. If the link of each $i$-simplex is (PL homeomorphic to) the sphere $S^{n-i-1}$ then the universal cover $\widetilde{\Delta}$ is an orientable PL $n$-manifold which therefore satisfies Poincaré duality: $H_{c}^{i}(\widetilde{\Delta}) \cong H_{n-i}(\widetilde{\Delta})$. The same holds if we only assume that the link of each $i$-simplex has the homology of $S^{n-i-1}$. In the case of interest in group theory, $\widetilde{\Delta}$ is assumed to be contractible, so that $\Delta$ is a finite $K(G, 1)$-complex, where $G=\pi_{1}(\Delta, v)$. Then Poincaré duality implies $H_{c}^{i}(\widetilde{\Delta}) \cong 0$ when $i \neq n$ and $H_{c}^{n}(\widetilde{\Delta}) \cong \mathbb{Z}$. This group $G$ is an example of an $n$-dimensional Poincaré duality group.

The motivation for this paper is the wish to provide an analogous set of statements for an $n$-dimensional Cohen-Macaulay complex, i.e., a connected finite $n$ dimensional simplicial complex $\Delta$ such that the link of each $i$-simplex has the homology of a non-trivial wedge of $(n-i-1)$-spheres. Again, in the case of interest in group theory, $\widetilde{\Delta}$ is assumed to be contractible, so that, again, $\Delta$ is a finite $K(G, 1)$ complex. The question is: What more must one know in order to deduce $H_{c}^{i}(\widetilde{\Delta}) \cong 0$ when $i \neq n$ and $H_{c}^{n}(\widetilde{\Delta})$ is torsion-free? If the answer is positive then $G$ is an $n$ dimensional duality group. For the definition of a duality group see [1] or [6]. (As the name implies, duality groups satisfy a generalization of Poincaré duality; we will have no need to make this duality explicit.) 
Remark. (1) The hypothesis implies $G$ is torsion-free and the requirement that $H_{c}^{0}(\widetilde{\Delta})=0$ is equivalent to $G$ being infinite. From now on, we assume $G$ is non-trivial.

(2) If an $n$-dimensional aspherical complex $\Delta$ satisfies $H_{c}^{i}(\widetilde{\Delta})=0$ when $i \neq n$, then it has to be the case that $H_{c}^{n}(\tilde{\Delta}) \neq 0$ (see e.g. Theorem 13.10 .1 of [6]), so that the geometric dimension of $G$ is $n$. From now on we assume the geometric dimension of $G$ is $n$.

Certainly, as we will see, further hypotheses are needed in order for $G$ to be a duality group.

1.2. The 2-dimensional case. In this paper we only discuss the case $n=2$. A connected finite 2-dimensional simplicial complex $\Delta$ is a 2-dimensional CohenMacaulay complex if the link of each vertex is non-empty and connected, and the link of each edge is non-empty. As before we write $G=\pi_{1}(\Delta, v)$ where $v$ is a vertex. Since we are assuming $H_{c}^{0}(\widetilde{\Delta})=0$ and $H_{c}^{2}(\widetilde{\Delta}) \neq 0$, the geometric dimension of $G$ must be 2. The question in Section 1.1 thus reduces to: When is it true that $H_{c}^{1}(\widetilde{\Delta})=0$ ? This is equivalent to asking: When does the 2-dimensional group $G$ have one end? It is a theorem of Stallings that a finitely generated torsion-free group has one end if and only if it does not decompose as a non-trivial free product. But we are seeking something different: we are assuming that nothing is known about the group $G$, and we wish to understand when we can deduce from the Cohen-Macaulay property that $\widetilde{\Delta}$ has one end. (The strictly analogous statement for a closed 2-manifold of positive genus is that, by Poincaré duality, its universal cover always has one end; the fact that one knows the homeomorphism type is a result of geometry rather than of algebraic topology.)

Our strategy is to first consider when a general contractible locally finite infinite 2-dimensional Cohen-Macaulay complex $X$ has one end. We will also require that the link of each vertex of $X$ is 2-connected graph, i.e., it is a connected graph and remains connected when the open star of any vertex is deleted. The main result of this paper is Theorem 5.6. This theorem looks complicated and has many hypotheses, but in the case where the complex $X$ is the universal cover of a connected finite 2-dimensional complex $\Delta$ it reduces to the following:

Theorem 1.1. Let $\Delta$ be a connected finite 2-dimensional aspherical Cohen-Macaulay complex such that the link of every vertex is 2-connected (in the sense of graph theory) and $\Delta$ does not consist of only one 2-simplex. Let $S_{0}$ be the singular set of $\Delta$ (i.e., the full subgraph generated by the edges which are faces of more than two 2-simplexes). If each component of the boundary of a regular neighborhood of $S_{0} \pi_{1}$-injects into $\Delta$, then $\widetilde{\Delta}$ has one end. In particular, $\pi_{1}(\Delta, v)$ is a 2-dimensional duality group.

That our task is non-trivial is shown by the following example:

Example 1.2 (Bestvina). Let $\Delta$ consist of two triangulated tori joined along a common edge. This complex is Cohen-Macaulay. The fundamental group of $\Delta$ is $\mathbb{Z}^{2} * \mathbb{Z}^{2}$ 
which is not a duality group ( $\widetilde{\Delta}$ is not one-ended). The links of the vertices of the common edge are two-petal roses, and the links of all other vertices are circles. Hence $\Delta$ is Cohen-Macaulay. The links of the vertices of the common edge are not 2-connected (if we remove the common vertex in the two-petal rose together with the edges that contain that vertex, the remaining part of the link is disconnected). The singular set $S_{0}$ in this complex consists only of one edge and neither component of $\partial N(S) \pi_{1}$-injects into $\Delta$.

Example 1.3. Let $\Delta$ consist of two triangulated tori joined along a common 2simplex. This complex is Cohen-Macaulay, and $\pi_{1}(\Delta) \cong \mathbb{Z}^{2} * \mathbb{Z}^{2}$, which is not one-ended. The link of each vertex is either a circle or the letter $\theta$. Hence the link of each vertex of $\Delta$ is 2 -connected. However, neither component of $\partial N(S) \pi_{1}$-injects into $\Delta$.

As a consequence of Theorem 1.1, we have Corollary 1.4 which provides necessary and sufficient conditions for $\widetilde{\Delta}$ to have one end. Let the singular set $S_{0}$ in $\Delta$ have components $S_{1}, S_{2}, \ldots, S_{n_{1}}$. We write $N=\bigsqcup_{i=1}^{n_{1}} N_{i}$ where the $N_{i}$ $\left(i=1,2, \ldots, n_{1}\right)$ are pairwise disjoint regular neighborhoods of $S_{1}, S_{2}, \ldots, S_{n_{1}}$ respectively. The boundary of $N$ in $\Delta$ consists of circles $C_{j}\left(j=1,2, \ldots, n_{2}\right)$. The closure in $\Delta$ of each component of $\Delta-N$ is a compact surface whose boundary consists of some of the circles $C_{j}$ (each circle $C_{j}$ occurs exactly once as a boundary of one surface). We denote these surfaces by $M_{k}\left(k=1,2, \ldots, n_{3}\right)$.

Corollary 1.4. Let $\Delta$ be a finite, 2-dimensional, aspherical, Cohen-Macaulay complex such that the link of each vertex of $\Delta$ is 2-connected and $\Delta$ does not consist of only one 2-simplex. Assume that no surface $M_{k}$ is a disk. Then the following are equivalent:

(i) Each $C_{j} \pi_{1}$-injects into $N$.

(ii) $\widetilde{\Delta}$ has one end and semistable fundamental group at infinity.

Remark. In [2], Brady, McCammond, and Meier used Morse theory to deal with the case when $\Delta$ is a finite, $n$-dimensional, non-positively curved complex. For any simplex $\sigma$, they defined a punctured link of $\sigma$ at $p$, denoted by $\operatorname{pkl}(\sigma, p)$, where $p$ is any point of $1 \mathrm{k} \sigma$, to be the set obtained by removing from $1 \mathrm{k} \sigma$ all points within $\frac{\pi}{2}$ of $p$. The punctured link of $\sigma$ is not in general a subcomplex of $1 \mathrm{k} \sigma$, but deformation retracts onto a maximal subcomplex of $1 \mathrm{k} \sigma$ that is contained in $\operatorname{plk}(\sigma, p)$. One of the results in [2] is: Let $\Delta$ be a finite, non-positively curved complex of dimension $n$. If for each cell $\sigma$ in $X$ and for each $p \in \operatorname{lk} \sigma$, the spaces $\operatorname{lk} \sigma$ and $\operatorname{plk}(\sigma, p)$ are $(n-\operatorname{dim} \sigma-2)$-acyclic, then $\pi_{1}(\Delta)$ is a duality group. (Corollary 1.4. in [2])

Acknowledgement. The results of this paper were part of my dissertation Groups of Geometric Dimension 2 written at Binghamton University. I would like to thank my advisor Ross Geoghegan for his support and help while working on the dissertation 
and this paper. His enthusiasm and encouragement made all the difference in my academic career. I would also like to thank Francisco J. Fernandez-Lasheras for helpful conversations during the preparation of the paper.

\section{Homology with coefficients in a local cohomology stack}

The exposition in this section is based on the work of E. C. Zeeman [12] and reworked in more detail by F. J. Fernandez-Lasheras in [5].

Let $X$ be an oriented, $n$-dimensional, locally finite, simplicial complex; this means that an orientation has been chosen for each simplex of $X$. Let $R$ be a ring.

We find it convenient to follow [6] (Section 12.1), calculating simplicial cohomology from chains (rather than cochains) and coboundaries.

The number $\left[\omega_{\beta}: \tau_{\alpha}\right]$, called the incidence number of the (oriented) simplices $\omega_{\beta}$ and $\tau_{\alpha}$, is defined as follows: If $\operatorname{dim} \tau_{\alpha}=i, \operatorname{dim} \omega_{\beta}=i+1, \omega_{\beta} \succ \tau_{\alpha}$, and $i \geq 1$, then $\left[\omega_{\beta}: \tau_{\alpha}\right]=1$ if the orientation of $\tau_{\alpha}$ inherited from $\omega_{\beta}$ agrees with the orientation of $\tau_{\alpha}$. Otherwise $\left[\omega_{\beta}: \tau_{\alpha}\right]=-1$. If $\tau_{\alpha}$ is not a simplex of $\omega_{\beta}$, then $\left[\omega_{\beta}: \tau_{\alpha}\right]=0$. This definition does not make sense for the case of edges and vertices. We will make the following convention: if $e$ is an (oriented) edge and $v$ is a vertex of $e$, then $[e: v]=1$ if $v$ is the terminal vertex of $e$, and $[e: v]=-1$ if $v$ is the initial vertex of $e$; if $v$ is not a vertex of $e$, then $[e: v]=0$.

Let $R(\tau)$ denote the free left $R$-module generated by the (oriented) simplex $\tau \in$ $X$. Let $C_{q}^{\infty}(X ; R)=\prod_{\operatorname{dim} \tau=q} R(\tau)$. Elements of $C_{q}^{\infty}(X ; R)$ are denoted by $\sum_{\operatorname{dim}\left(\tau_{\alpha}\right)=q} m_{\alpha} \tau_{\alpha}$ where $m_{\alpha} \in R$, and they are called infinite simplicial $q$-chains (or locally finite q-chains) in $X$ with coefficients in $R$. The coboundary homomorphism for infinite chains is $\partial: C_{q}^{\infty}(X ; R) \rightarrow C_{q+1}^{\infty}(X ; R)$ defined by

$$
\delta\left(\sum_{\alpha} m_{\alpha} \tau_{\alpha}\right)=\sum_{\beta}\left(\sum_{\alpha} m_{\alpha}\left[\omega_{\beta}: \tau_{\alpha}\right]\right) \omega_{\beta}
$$

where $\operatorname{dim} \tau_{\alpha}=q$ and $\operatorname{dim} \omega_{\beta}=q+1$.

For oriented simplicial pairs $(X, A)$ let

$$
\begin{array}{r}
C_{q}^{\infty}(X, A ; R):=\left\{\sum m_{\alpha} \tau_{\alpha} \in C_{q}^{\infty}(X ; R): m_{\alpha}=0 \text { whenever } \tau_{\alpha}\right. \text { is } \\
\text { a } q \text {-simplex of } A\} .
\end{array}
$$

The relative cohomology modules $H^{*}(X, A ; R)$ are calculated from the cochain complex $\left(C_{*}^{\infty}(X, A ; R), \delta\right)$. Of course $H^{q}(X, A ; R)=Z^{q}(X, A ; R) / B^{q}(X, A ; R)$, where

$$
\begin{array}{r}
Z^{q}(X, A ; R)=\left\{c \in C_{q}^{\infty}(X ; R): \delta c=0 \text { and the coefficient in } c\right. \text { corresponding } \\
\text { to each } q \text {-simplex of } A \text { is } 0\}
\end{array}
$$


and

$$
B^{q}(X, A ; R)=\left\{c \in C_{q}^{\infty}(X ; R): c=\delta d \quad \text { where } d \in C_{q-1}^{\infty}(X, A ; R)\right\} .
$$

This way of defining $H^{*}(X, A ; R)$ can be found in [6] (Chapter 12).

The number of ends of $X$ does not depend of the ring $R$ (this follows from [6] Theorem 13.5.5). Therefore, it is enough to work with $\mathbb{Z}_{2}$-coefficients and from now on $R=\mathbb{Z}_{2}$.

By $\mathfrak{D}(X)$ we will denote the following category: the objects are the simplices $\sigma \in X$, and a morphism $\tau \rightarrow \sigma$ is the relation $\tau \succ \sigma$. The star of $\sigma \in X$ is the set st $_{X} \sigma=\{\tau \in X: \tau \cup \sigma \in X\}$, the link of $\sigma$ in $X$ is the set $\operatorname{lk}_{X} \sigma=\left\{\tau \in\right.$ st $_{X} \sigma$ : $\tau \cap \sigma=\emptyset\}$, and the open star of $\sigma$ in $X$ is the set st $_{X} \sigma=\{\tau \in X: \tau \succ \sigma\}$. We often omit the index $X$. Note that $\mathrm{lk} \sigma$ and st $\sigma$ are subcomplexes of $X$, and $\stackrel{\circ}{\text { st } \sigma \text { is }}$ not (in general).

Definition 2.1. The $q$ th-local cohomology stack on $X, q \geq 0$ is the covariant functor $\mathcal{L}^{q}: \mathfrak{D}(X) \rightarrow$ Abelian $\mathcal{G}$ roups defined as follows:

$$
\mathcal{L}^{q}(\sigma)=H^{q}(X, X-\stackrel{\circ}{\operatorname{st}}(\sigma))
$$

where $\sigma$ is a simplex of $X$. If $\sigma$ is a simplex of $\tau$, i.e., if there is a morphism $\tau \rightarrow \sigma$, then $\left(\mathcal{L}^{q}\right)^{\tau, \sigma}: \mathcal{L}^{q}(\tau) \rightarrow \mathcal{L}^{q}(\sigma)$ is induced by the inclusion $\sigma \subseteq \tau$.

Lemma 2.2. If $\sigma$ is a simplex of $X$ then $H^{q}(X, X-\stackrel{\circ}{\mathrm{st}} \sigma) \cong \tilde{H}^{q-\operatorname{dim} \sigma-1}(\operatorname{lk} \sigma)$.

Proof. First we note that $(X-\stackrel{\circ}{\text { st }} \sigma) \cap$ st $\sigma$ is homeomorphic to bd $\sigma * 1 \mathrm{k} \sigma$, which is homeomorphic to $\sum^{\operatorname{dim} \sigma}(\operatorname{lk} \sigma)$, where $\sum^{\operatorname{dim} \sigma}$ stands for the $(\operatorname{dim} \sigma)^{\text {th }}$-suspension of $\sigma$. Then, by excision, we have

$$
\left.H^{q}(X, X-\stackrel{\circ}{\text { st } \sigma})\right) \cong H^{q}(\text { st } \sigma,(X-\stackrel{\circ}{\text { st } \sigma}) \cap \text { st } \sigma) \cong H^{q}\left(\text { st } \sigma, \sum^{\operatorname{dim} \sigma}(\operatorname{lk} \sigma)\right) .
$$

Since st $\sigma$ is contractible, we have

$$
\left.H^{q}(X, X-\stackrel{\circ}{\mathrm{st} \sigma})\right) \cong H^{q-1}\left(\sum^{\operatorname{dim} \sigma}(\operatorname{lk} \sigma)\right) \cong \tilde{H}^{q-\operatorname{dim} \sigma-1}(\operatorname{lk} \sigma)
$$

Corollary 2.3. If $X$ is an $n$-dimensional Cohen-Macaulay complex then $\mathcal{L}^{q}(\sigma) \cong 0$ for $q<n$ for any simplex $\sigma \in X$.

Proof. If $\sigma$ is a simplex of $X$, then $\tilde{H}^{q-\operatorname{dim} \sigma-1}(\mathrm{lk} \sigma) \cong 0$ when $q-\operatorname{dim} \sigma-1<$ $\operatorname{dim} \operatorname{lk} \sigma$. Since $X$ is pure, i.e., all of its maximum faces have dimension $\operatorname{dim} X$, it follows that $\operatorname{dim}(\mathrm{lk} \sigma)=\operatorname{dim} X-\operatorname{dim} \sigma-1$ ([4], Corollary 5.1.5, page 210), which implies $\widetilde{H}^{q-\operatorname{dim} \sigma-1}(\mathrm{lk} \sigma) \cong 0$ when $q<\operatorname{dim} 1 \mathrm{k} \sigma+\operatorname{dim} \sigma+1=n$. Therefore $\mathcal{L}^{q}(\sigma)=H^{q}(X, X-$ st $\sigma) \cong 0$ if $q<n$. 
We define a chain complex $J_{*}\left(X, \mathcal{L}^{n}\right)$ as follows:

Let

$$
J_{q}\left(X, \mathcal{L}^{n}\right):=\bigoplus_{\operatorname{dim} \sigma=q} \mathcal{L}^{n}(\sigma), \quad q \geq 0 .
$$

Define $\partial_{\mathcal{L}}: J_{q}\left(X, \mathcal{L}^{n}\right) \rightarrow J_{q-1}\left(X, \mathcal{L}^{n}\right)$ as follows:

$$
\partial_{\mathcal{L}}\left(\sum_{i=1}^{m} a_{i} h_{i}\right)=\sum_{i=1}^{m} \sum_{\tau_{i} \succ \sigma} a_{i}\left[\tau_{i}: \sigma\right]\left(\mathcal{L}^{q}\right)^{\tau_{i}, \sigma}\left(h_{i}\right)
$$

where $h_{i} \in \mathcal{L}^{q}\left(\tau_{i}\right), \sum_{i=1}^{m} a_{i} h_{i} \in J_{q}\left(X, \mathcal{L}^{n}\right), \operatorname{dim}\left(\tau_{i}\right)=q, \operatorname{dim}(\sigma)=q-1$. Then $\left(J_{q}\left(X, \mathcal{L}^{n}\right) ; \partial_{\mathcal{L}}\right)$ is a chain complex. We denote its homology by $H_{q}\left(X, \mathcal{L}^{n}\right)$.

In the spirit of Zeeman [12], Lasheras [5] has proved the following generalization of Poincaré Duality:

Theorem 2.4. Let $X$ be an $n$-dimensional, locally finite, Cohen-Macaulay simplicial complex. Then for all $p \in \mathbb{Z}$

$$
H_{p}\left(X, \mathcal{L}^{n}\right) \cong H_{c}^{n-p}(X) .
$$

In Zeeman [12] there is a version of this theorem when $X$ is finite complex.

\section{About $H_{*}\left(X, \mathcal{L}^{2}\right)$}

Let $X$ be an oriented, 2-dimensional, path connected, locally finite Cohen-Macaulay complex. We will present an alternative way of viewing $H_{*}\left(X, \mathcal{L}^{2}\right)$.

Let $K_{2}, K_{1}$, and $K_{0}$ be the free abelian groups generated by all pairs $(\sigma, \sigma),(\sigma, e)$, and $(\sigma, v)$ respectively, where $\sigma$ is a 2-simplex of $X, e$ is an edge of $\sigma$ and $v$ is a vertex of $\sigma$. Then $\left(K_{*}, \partial^{\prime}\right)$ is a chain complex, where the boundary is defined as follows:

$$
\begin{aligned}
\partial^{\prime}(\sigma, \sigma) & =(\sigma, \partial \sigma):=\left(\sigma, e_{1}\right)+\left(\sigma, e_{2}\right)+\left(\sigma, e_{3}\right), \\
\partial^{\prime}(\sigma, e) & =(\sigma, \partial e):=\left(\sigma, v_{1}\right)+\left(\sigma, v_{2}\right) .
\end{aligned}
$$

Here, $\partial$ is the boundary homomorphism for simplicial homology, $\partial \sigma=\left[\sigma: e_{1}\right] e_{1}+$ $\left[\sigma: e_{2}\right] e_{2}+\left[\sigma: e_{3}\right] e_{3}$, and $\partial e=\left[e: v_{1}\right] v_{1}+\left[e: v_{2}\right] v_{2}$.

We will use the following:

$$
(\delta e, e):=\sum_{\sigma \succ e}[\sigma: e](\sigma, e), \quad(\delta e, v):=\sum_{\sigma \succ e}[\sigma: e](\sigma, v) .
$$

Here $\left(J_{*}, \partial_{\mathcal{L}}\right)$ is the chain complex from Section 2 used to define $H_{*}\left(X, \mathcal{L}^{2}\right)$. If $[\omega] \in J_{i}=\bigoplus_{\operatorname{dim} \tau=i} H^{2}(X, X-\stackrel{\circ}{\text { st } \tau})$ we denote the $\tau$-component by $[\omega]_{\tau} \in$

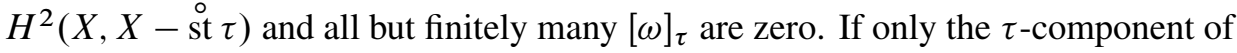
$[\omega]$ is non-zero we abuse notation by writing $[\omega]_{\tau} \in J_{i}$. 
First we will see how $\partial_{\mathcal{L}}$ works. Let $[\sigma]_{\sigma} \in J_{2}$ where $\sigma$ is a 2-simplex. Using the definition for $\partial_{\mathcal{L}}$ we have

$$
\partial_{\mathcal{L}}\left([\sigma]_{\sigma}\right)=\sum_{i=1}^{3}\left[\sigma: e_{i}\right][\sigma]_{e_{i}},
$$

where $e_{1}, e_{2}$, and $e_{3}$ are the edges of $\sigma$.

Let $[\sigma]_{e} \in J_{1}$ where $\sigma$ is a 2 -simplex and $e$ is an edge of $\sigma$. Then

$$
\partial_{\mathcal{L}}\left([\sigma]_{e}\right)=\left[\sigma: v_{1}\right][\sigma]_{v_{1}}+\left[\sigma: v_{2}\right][\sigma]_{v_{2}},
$$

where $v_{1}$, and $v_{2}$ are vertices of $e$.

The projection maps $\pi_{i}: K_{i} \rightarrow J_{i}$ are defined by $\pi_{i}(\sigma, \tau)=[\sigma]_{\tau}, i=0,1,2$. Let $L_{i}:=\operatorname{ker} \pi_{i}$. Then $\left(L_{*}, \partial^{\prime}\right)$ is a chain complex.

We will consider the following diagram:

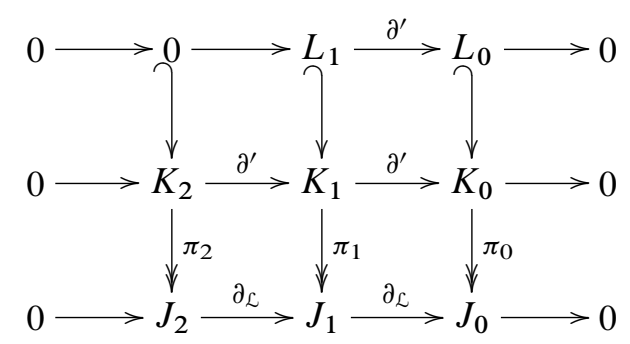

Figure 1

The columns are exact. The rows are chain complexes. We must show that the diagram commutes.

We will give a simpler description of the abelian groups $L_{1}$ and $L_{2}$.

Lemma 3.1. The abelian groups $L_{0}$ and $L_{1}$ are generated by all pairs $(\delta e, v)$ and $(\delta e, e)$ respectively, where $e$ is an edge of $X$ and $v$ is a vertex of $e$; and $L_{2} \cong 0$.

Proof. First we will show that $L_{2} \cong 0$ by showing that ker $\pi_{2}=0$.

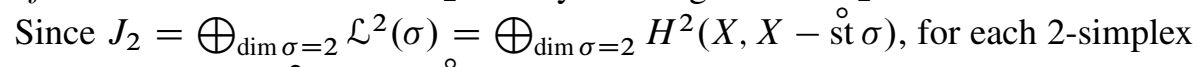
$\sigma$ we will calculate $H^{2}(X, X-\stackrel{\circ}{\text { st } \sigma)}$ from the following cochain complex:

$$
0 \rightarrow C_{0}^{\infty}\left(X, X-\stackrel{\circ}{\text { st } \sigma)} \rightarrow C_{1}^{\infty}(X, X-\stackrel{\circ}{\text { st } \sigma}) \rightarrow C_{2}^{\infty}(X, X-\stackrel{\circ}{\text { st } \sigma}) \rightarrow 0 ;\right.
$$

$C_{2}^{\infty}\left(X, X-\stackrel{\circ}{\text { st } \sigma)}\right.$ is generated by $\sigma$, and $C_{1}^{\infty}\left(X, X-\stackrel{\circ}{\text { st } \sigma)}=0\right.$. Hence $H^{2}(X, X-$ st $\sigma)$ is generated by $[\sigma]$, i.e., $H^{2}(X, X-$ st $\sigma) \cong \mathbb{Z}_{2}$. Let $x:=\sum_{\sigma \in X} m_{\sigma}(\sigma, \sigma) \in$ 
$K_{2}$. Then $\pi_{2}(x)=\sum_{\sigma \in X} m_{\sigma}\left([\sigma]_{\sigma}\right)$ and $\pi_{2}(x)=0$ if and only if $m_{\sigma}=0$ for all 2-simplices $\sigma$. Hence, $\operatorname{ker} \pi_{2}=0$, and therefore $L_{2} \cong 0$.

Next we will consider $J_{1}=\bigoplus_{\operatorname{dim} e=1} \mathcal{L}^{2}(e)=\bigoplus_{\operatorname{dim} e=1} H^{2}(X, X-$ st $e$ ). For each edge $e$, we will calculate $H^{2}(X, X-$ st $e)$ from the cochain complex

$$
0 \rightarrow C_{0}^{\infty}(X, X-\stackrel{\circ}{\text { st }} e) \rightarrow C_{1}^{\infty}(X, X-\stackrel{\circ}{\text { st }} e) \rightarrow C_{2}^{\infty}(X, X-\stackrel{\circ}{\text { st } e}) \rightarrow 0 .
$$

The abelian group $C_{2}^{\infty}(X, X-$ st $e)$ is generated by all 2-simplices $\sigma$ such that $e$ is an edge of $\sigma$. For each edge $e$, the free abelian group $C_{1}^{\infty}(X, X-$ st $e)$ is generated by $e$. Let $x=\sum m_{\sigma, e}(\sigma, e)$. Then $\pi_{1}(x)=\sum m_{\sigma, e}\left([\sigma]_{e}\right)$. For each edge $e$ that appears in $x$, we will define $x_{e}$ to be the sum of those pairs from $x$ that have second coordinate $e$, i.e., $x_{e}:=\sum_{\sigma} m_{\sigma, e}(\sigma, e)$. Then $\pi_{1}\left(x_{e}\right)=\sum_{\sigma} m_{\sigma, e}[\sigma]_{e}$. Hence $\pi_{1}\left(x_{e}\right)=[0]$ if and only if $\sum_{\sigma} m_{\sigma, e}[\sigma]_{e}=[0]$ (this summation is in $H^{2}(X, X-$ st $e$ )). So $\sum_{\sigma} m_{\sigma, e}[\sigma]_{e}=[0]$ if and only if there is a constant $m_{e}$ such that $\sum_{\sigma \succ e} m_{\sigma, e} \sigma=$ $m_{e} \delta e$. Therefore, $x_{e} \in \operatorname{ker} \pi_{1}$ if and only if $x_{e}=\sum_{\sigma \succ e} m_{\sigma, e}(\sigma, e)=m_{e}(\delta e, e)$. Since $x=\sum_{e} x_{e}$, and $\pi_{1}(x)=\sum_{e} \pi_{1}\left(x_{e}\right)$, it follows that the elements of ker $\pi_{1}$ are finite sums of pairs of type $(\delta e, e)$, i.e., $L_{1}$ is generated by all pairs $(\delta e, e)$ where $e$ is an edge of $X$.

Similarly we can show that $L_{0}$ is generated by all pairs $(\delta e, v)$ where $v$ is a vertex of $X$ and $e$ has $v$ as a vertex.

Using Lemma 3.1 it is straightforward to show that the diagram commutes. We omit the details.

Proposition 3.2. $H_{1}\left(K, \mathbb{Z}_{2}\right) \cong 0$.

Proof. Let $\beta=\sum_{\sigma \succ e} m_{(\sigma, e)}(\sigma, e) \in Z_{1}(K)$, i.e., $\partial^{\prime} \beta=0$ (only a finite number of coefficients $m_{\sigma, e}$ are 1). Let $m_{\sigma, e}=1$ for some 2-simplex $\sigma$ and some edge $e$ of $\sigma$. Since $\partial^{\prime}(\sigma, e)=\left(\sigma, v_{1}\right)+\left(\sigma, v_{2}\right)$ where $v_{1}$ and $v_{2}$ are vertices of $e$, we know that $\left(\sigma, v_{1}\right)$ and $\left(\sigma, v_{2}\right)$ are present (i.e., have non-zero coefficient) in $\partial^{\prime} \beta$. We will denote by $e_{1}$ and $e_{2}$ the other two edges of $\sigma$.

The only way to cancel $\left(\sigma, v_{1}\right)$ is if there is another term $\left(\sigma, v_{1}\right)$ in $\partial^{\prime} \beta$. We will get another term $\left(\sigma, v_{1}\right)$ only if $\left(\sigma, e_{1}\right)$ is present in $\beta$. Similarly, the only way to cancel $\left(\sigma, v_{2}\right)$ is if $\left(\sigma, e_{2}\right)$ is present in $\beta$. Hence, in $\beta$ we have the following three terms $(\sigma, e),\left(\sigma, e_{1}\right)$, and $\left(\sigma, e_{2}\right)$ with coefficient 1 . But $(\sigma, e)+\left(\sigma, e_{1}\right)+\left(\sigma, e_{2}\right)=\partial^{\prime}(\sigma, \sigma)$. So $\beta \in B_{1}(K)$, i.e., each cycle in $K_{1}$ bounds. Therefore $H_{1}\left(K, \mathbb{Z}_{2}\right) \cong 0$.

Theorem 3.3. If $X$ is an infinite, 2-dimensional, path-connected, Cohen-Macaulay complex then $H_{1}\left(X, \mathcal{L}_{\mathbb{Z}_{2}}^{2}\right) \cong \operatorname{ker}\left\{H_{0}\left(L, \mathbb{Z}_{2}\right) \rightarrow H_{0}\left(K, \mathbb{Z}_{2}\right)\right\}$.

Proof. From the short exact sequence of chain complexes (Figure 1) we have the 
following long exact sequence:

$$
\begin{aligned}
0 \longrightarrow & H_{2}\left(L, \mathbb{Z}_{2}\right) \longrightarrow H_{2}\left(K, \mathbb{Z}_{2}\right) \longrightarrow H_{2}\left(J, \mathbb{Z}_{2}\right) \\
& \longrightarrow H_{1}\left(L, \mathbb{Z}_{2}\right) \longrightarrow H_{1}\left(K, \mathbb{Z}_{2}\right) \longrightarrow H_{1}\left(J, \mathbb{Z}_{2}\right) \\
& \longrightarrow H_{0}\left(L, \mathbb{Z}_{2}\right) \longrightarrow H_{0}\left(K, \mathbb{Z}_{2}\right) \longrightarrow H_{0}\left(J, \mathbb{Z}_{2}\right) \longrightarrow 0
\end{aligned}
$$

By Proposition 3.2 we have $H_{1}\left(K, \mathbb{Z}_{2}\right) \cong 0$. Hence, we have the following long exact sequence

$$
0 \longrightarrow H_{1}\left(J, \mathbb{Z}_{2}\right) \longrightarrow H_{0}\left(L, \mathbb{Z}_{2}\right) \longrightarrow H_{0}\left(K, \mathbb{Z}_{2}\right) \longrightarrow H_{0}\left(J, \mathbb{Z}_{2}\right) \longrightarrow 0 .
$$

Therefore $H_{1}\left(X, \mathcal{L}_{\mathbb{Z}_{2}}^{2}\right)=H_{1}\left(J, \mathbb{Z}_{2}\right) \cong \operatorname{ker}\left\{H_{0}\left(L, \mathbb{Z}_{2}\right) \rightarrow H_{0}\left(K, \mathbb{Z}_{2}\right)\right\}$.

Because of Theorem 2.4 this gives a characterization of $H_{c}^{1}\left(X ; \mathbb{Z}_{2}\right)$, i.e., the number of ends of $X$.

\section{2-connected links}

We are assuming that $X$ is an infinite, oriented, 2-dimensional, path connected CohenMacaulay complex. From now we also assume that $X$ is acyclic with respect to $\mathbb{Z}_{2}$-coefficients, i.e., $\widetilde{H}_{i}\left(X ; \mathbb{Z}_{2}\right) \cong 0$ for all integers $i$.

Our goal now is to determine what additional conditions on $X$ are sufficient to ensure $H_{c}^{1}\left(X, \mathbb{Z}_{2}\right) \cong 0$, or, using Theorem 2.4, to ensure $H_{1}\left(X, \mathcal{L}_{\mathbb{Z}_{2}}^{2}\right) \cong 0$. This is equivalent to " $X$ has one end".

Let $\Gamma$ be a graph with at least three vertices and let $w$ be a vertex of $\Gamma$. We say that a vertex $w \in \Gamma$ is a cut vertex of $\Gamma$ if the removal of $w$ and all edges containing $w$ causes an increase in the number of connected components of $\Gamma$. A graph $\Gamma$ that does not have a cut vertex is called a 2-connected graph. In Example 1.2, the links of the vertices $v_{1}$ and $v_{2}$ are not 2-connected and the links of all other vertices are 2-connected.

Lemma 4.1. If the links of all vertices of $X$ are 2-connected, then each edge of $X$ is a face of at least two 2-simplices.

Proof. Assume that there is an edge of $X$, denoted by $e_{1}$, that is a face of only one 2 -simplex. We denote by $\sigma$ the 2 -simplex that has $e_{1}$ as an edge, we denote the vertices of $\sigma$ by $v_{1}, v_{2}$, and $v_{3}$, and the other two edges by $e_{2}$ and $e_{3}$, where $v_{i}$ is the vertex opposite $e_{i}$ in $\sigma$. Then $e_{3}$ is the only edge in $1 \mathrm{k} v_{3}$ that has $v_{2}$ as a vertex, and $v_{1}$ is also a vertex of that edge. If $e_{2}$ is a face of at least two 2-simplices, then if we remove the vertex $v_{1}$ from $l \mathrm{k} v_{3}$ together with the edges that have $v_{1}$ as a vertex, the remaining part of $l \mathrm{k} v_{3}$ will not be connected; the vertex $v_{2}$ is not connected to the remaining part of the link. Hence $v_{1}$ is a cut vertex, but this is not possible because the link of each vertex of $X$ is 2-connected (i.e., does not have any cut vertices). 
Therefore $e_{2}$ is an edge of no other 2-simplex besides $\sigma$. Similarly we can conclude that $e_{3}$ is an edge of no other 2-simplex besides $\sigma$. Hence $X$ consists of only one 2-simplex which is a contradiction since $X$ is infinite and path connected.

If $X$ is a finite simplicial complex, from the proof of previous lemma we have the following corollary.

Corollary 4.2. If $X$ is a finite, path connected simplicial complex and the links of all vertices of $X$ are 2-connected, then each edge of $X$ is a face of at least two 2-simplices or $X$ consists of only one 2-simplex.

Now we will discuss the impact that the new condition "the link of each vertex of $X$ is 2-connected" has on the structure of the complex $X$. We will consider once again the diagram in Figure 1. By Theorem 3.3 we are interested in $\operatorname{ker}\left\{H_{0}\left(L, \mathbb{Z}_{2}\right) \rightarrow\right.$ $\left.H_{0}\left(K, \mathbb{Z}_{2}\right)\right\} \cong 0$.

We will find necessary and sufficient conditions (Theorem 4.5) for a chain $\alpha$ in $K_{0}$ to be in $L_{0}$.

Let $\alpha=\sum_{k=1}^{m} \sum_{\sigma_{i} \succ v_{k}} n_{i k}\left(\sigma_{i}, v_{k}\right)$. For each vertex $v_{k}$ we define

$$
\alpha_{k}:=\sum_{\sigma_{i} \succ v_{k}} n_{i k}\left(\sigma_{i}, v_{k}\right) .
$$

It follows that $\alpha=\sum_{k} \alpha_{k}$.

Since $X$ is 2-dimensional Cohen-Macaulay complex, the link of each vertex $v_{k}$ is a connected graph. Let $e$ be an arbitrary edge of lk $v_{k}$. Let $\sigma_{i}$ be the 2-simplex of st $v_{k}$ that has $e$ as a face (this 2 -simplex is unique). We will relabel the edges from lk $v_{k}$ in the following way: we will label the edge of $\sigma_{i}$ opposite $v_{k}$ by $e_{i k}$. Let

$$
\bar{\alpha}_{k}:=\sum_{\sigma_{i} \succ v_{k}} n_{i k} e_{i k} .
$$

The difference between $\alpha_{k}$ and $\bar{\alpha}_{k}$ is that $\left(\sigma_{i}, v_{k}\right)$ is replaced by $e_{i k}$.

Let $v$ and $w$ be two vertices in $X$. A path joining $v$ and $w$ is a sequence of distinct vertices (except that $v$ may equal $w$ ) $\gamma: v=v_{0}, v_{1}, v_{2}, \ldots, v_{n-1}, v_{n}=w$ such that any two consecutive vertices are joined by an edge in $X$. If $v=w$, then the path is a cycle.

The proof of the following proposition can be found in [3] (Theorem 13.5.3).

Proposition 4.3. The link of each vertex of $X$ is 2-connected if and only if for each vertex $v \in X$, the link of $v$ has the following property: for any two edges $e$ and $f$ of $1 \mathrm{k} v$ there is a cycle in $\mathrm{lk} v$ that contains $e$ and $f$.

Lemma 4.4. Let $\alpha=\sum_{k=1}^{m} \sum_{\sigma_{i} \succ v_{k}} n_{i k}\left(\sigma_{i}, v_{k}\right) \in K_{0}$. Then $\alpha \in L_{0}$ if and only if for each vertex $v_{k}$ that appears in $\alpha, \bar{\alpha}_{k}$ is a (1-dimensional) coboundary in $1 \mathrm{k} v_{k}$. 
Proof. Suppose that $\alpha \in L_{0}$. Then

$$
\alpha=\sum_{k=1}^{m} \sum_{e_{j} \succ v_{k}} n_{j k}^{\prime}\left(\delta e_{j}, v_{k}\right)
$$

where $n_{i k}=n_{j_{1} k}^{\prime}+n_{j_{2} k}^{\prime}$, and $e_{j_{1}}$ and $e_{j_{2}}$ are the edges of $\sigma_{i}$ that have $v_{k}$ as a vertex. For fixed $v_{k}, \bar{\alpha}_{k}=\sum_{k=1}^{m} \sum_{e_{j} \succ v_{k}} n_{j k}^{\prime} \delta_{\mathrm{lk} v_{k}} v_{j k}$, where $v_{j k}$ is the vertex of $e_{j}$ different from $v_{k}$, and $\delta_{\mathrm{lk}} v_{k}$ denotes the coboundary in the cochain complex of lk $v_{k}$. Hence $\bar{\alpha}_{k}$ is a coboundary in lk $v_{k}$.

Suppose that $\bar{\alpha}_{k}$ is 1-dimensional coboundary in $1 \mathrm{k} v_{k}$ for each vertex $v_{k}$ that appears in $\alpha$. Then, for fixed $v_{k}, \bar{\alpha}_{k}=\sum_{k=1}^{m} \sum_{e_{j} \succ v_{k}} n_{j k}^{\prime} \delta_{\mathrm{lk} v_{k}} v_{j k}$. Hence, for fixed $v_{k}, \alpha_{k}=\sum_{k=1}^{m} \sum_{e_{\succ} v_{k}} n_{j k}^{\prime}\left(\delta e_{j}, v_{k}\right)$ which is in $L_{0}$. Since $\alpha=\sum_{k} \alpha_{k}$, it follows that $\alpha \in L_{0}$.

Theorem 4.5. Let the link of each vertex of $X$ be 2-connected. Then $\alpha \in L_{0}$ if and only if for each $v_{k}$ that appears in $\alpha$ and each cycle $\gamma$ of $1 \mathrm{k} v_{k}$ the sum of the coefficients of $\bar{\alpha}_{k}$ corresponding to the edges of $\gamma$ is 0 .

Proof. Suppose that $\alpha \in L_{0}$, i.e., $\alpha=\sum_{k=1}^{m} \sum_{\sigma_{i} \succ v_{k}} n_{i k}\left(\sigma_{i}, v_{k}\right)$. Let $v_{k}$ be a vertex appearing in $\alpha$. By Lemma $4.4, \bar{\alpha}_{k}$ is a 1 -dimensional coboundary in lk $v_{k}$. Hence $\bar{\alpha}_{k}=\sum_{k=1}^{m} \sum_{e_{j} \succ v_{k}} n_{j k}^{\prime} \delta_{\mathrm{lk} v_{k}} v_{j k}$, where $v_{j k}$ is the vertex of $e_{j}$ different from $v_{k}$. Let $\gamma$ be an arbitrary cycle of lk $v_{k}$. If a vertex $v_{j k}$ that appears in $\bar{\alpha}_{k}$ lies on $\gamma$, then there are exactly two edges from $\gamma$ that have $v_{j k}$ as a vertex. Hence, the sum of the coefficients corresponding to those two edges in $\bar{\alpha}_{k}$ is 0 . Therefore, for each vertex of $\gamma$ that appears in $\bar{\alpha}_{k}$ we have exactly two edges of $\gamma$ that have coefficient 1 in $\bar{\alpha}_{k}$. So the sum of the coefficients of $\bar{\alpha}_{k}$ corresponding to the edges of $\gamma$, and therefore on each cycle of $\mathrm{lk} v_{k}$, is 0 . This proves "only if".

Suppose now that, for each vertex $v_{k}$ that appears in $\alpha$, the sum of the coefficients from $\bar{\alpha}_{k}=\sum n_{i k} e_{i k}$ that correspond to the edges that lie on each cycle of $1 \mathrm{k} v_{k}$ is 0 . We will pick an edge from $\bar{\alpha}_{k}$ with non-zero coefficient, denoted by $e_{0}$ (the edges of $X$ are already labeled; here we relabel them just for the purpose of this proof). Denote the vertices of $e_{0}$ by $v_{0}$ and $v_{1}$. Let $\gamma_{0}, \gamma_{1}, \ldots, \gamma_{s}$ be all the cycles starting and ending at $v_{0}$ and containing $e_{0}$. The graph $1 \mathrm{k} v_{k}$ is covered by these cycles, i.e., every edge of $1 \mathrm{k} v_{k}$ lies on at least one cycle $\gamma_{i}, i=0,1,2, \ldots, s$ (this follows from Proposition 4.3). We will show that $\bar{\alpha}_{k}$ is a 1-dimensional coboundary in lk $v_{k}$ by rewriting it using the cycles $\gamma_{0}, \gamma_{1}, \ldots, \gamma_{s}$. We will start with the cycle $\gamma_{0}$ and we will write the terms from $\bar{\alpha}_{k}$ whose edges are on $\gamma_{0}$ as a coboundary in $\gamma_{0}$.

Let $e_{0}, e_{1}, e_{2}, \ldots, e_{t\left(\gamma_{0}\right)}$ be the edges of $\gamma_{0}$ and $v_{0}, v_{1}, v_{2}, \ldots, v_{t\left(\gamma_{0}\right)}$ be the vertices of $\gamma_{0}$ (as in Figure 2). If $e_{1}$ is present in $\bar{\alpha}_{k}$, then we can write $e_{0}+e_{1}$ as $\delta_{\gamma_{0}} v_{1}$. If $e_{1}$ is not present in $\bar{\alpha}_{k}$, then we can add $e_{1}+e_{1}$ to $\bar{\alpha}_{k}$ and we will have the previous case. Then we will consider the edge $e_{2}$, and "play the same trick" as with $e_{1}$, and so on. Since the sum of the coefficients of $\bar{\alpha}_{k}$ corresponding to $\gamma_{0}$ is 0 , we can write 


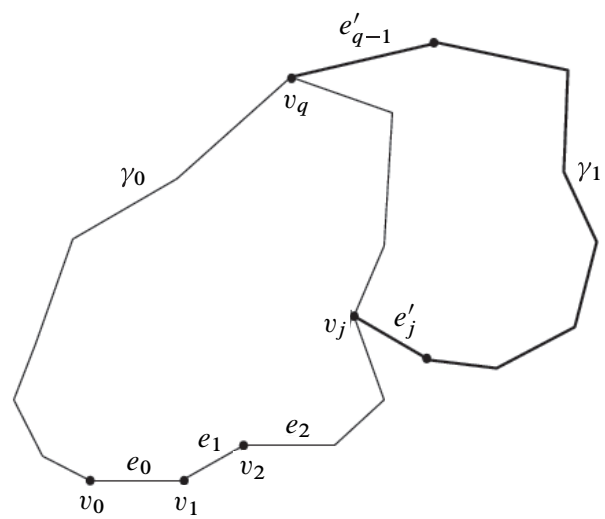

Figure 2

the terms of $\bar{\alpha}_{k}$ that correspond to edges of $\gamma_{0}$ as a coboundary of $\gamma_{0}$, i.e.,

$$
\bar{\alpha}_{k}=\sum_{v \in \gamma_{0}} \delta_{\gamma_{0}} v+\left(\text { the rest of } \bar{\alpha}_{k}\right) \text {. }
$$

We will repeat the same process with $\gamma_{1}$ but our goal now is to write the terms of $\bar{\alpha}_{k}$ corresponding to $\gamma_{0}$ and $\gamma_{1}$ as a coboundary of $\gamma_{0} \cup \gamma_{1}$. The cycle $\gamma_{1}$ has common edges with $\gamma_{0}$ (at least $e_{0}$ is a common edge). So we will start with the first term from $\bar{\alpha}_{k}$ that lies on $\gamma_{1}$ but not on $\gamma_{0}$. We will use the notation of Figure 2. If $\delta_{\gamma_{0}} v_{j}$ is not in $\bar{\alpha}_{k}$ then we will pick the first edge from $\gamma_{1}$ moving along $\gamma_{1}$ counterclockwise that has a non-zero coefficient in $\bar{\alpha}_{k}$ and using the same process as with the cycle $\gamma_{0}$ we will write $\bar{\alpha}_{k}=\sum_{v \in \gamma_{0} \cup \gamma_{1}} \delta_{\gamma_{0} \cup \gamma_{1}} v+\left(\right.$ the rest of $\bar{\alpha}_{k}$ ). If $\delta_{\gamma_{0}} v_{j}$ is in $\bar{\alpha}_{k}$, then we have two possibilities: either $e_{j}^{\prime}$ is in $\bar{\alpha}_{k}$ or not. If $e_{j}^{\prime}$ is in $\bar{\alpha}_{k}$, then combining $e_{j}^{\prime}$ with $\delta_{\gamma_{0}} v_{j}$ we will get a term $\delta_{\gamma_{0} \cup \gamma_{1}} v_{j}$ in $\bar{\alpha}_{k}$. If $e_{j}^{\prime}$ is not in $\bar{\alpha}_{k}$, then we will add $e_{j}^{\prime}+e_{j}^{\prime}$ to $\bar{\alpha}_{k}$ and get the case when $e_{j}^{\prime}$ is in $\bar{\alpha}_{k}$. We will continue "moving" along $\gamma_{1}$ and rewriting the terms of $\bar{\alpha}_{k}$. We need to consider a case when $\gamma_{1}$ meets $\gamma_{0}$ again. Let $v_{q}$ be a vertex where $\gamma_{0}$ and $\gamma_{1}$ meet again. We must rule out the possibility that $\delta_{\gamma_{0}} v_{q}$ is in $\bar{\alpha}_{k}$ but $e_{q-1}^{\prime}$ is not (or vice versa). Consider the cycle that contains the parts from $\gamma_{0}$ and $\gamma_{1}$ between $v_{j}$ and $v_{q}$. The sum of the coefficients of $\bar{\alpha}_{k}$ corresponding to this cycle is 0 . Therefore $\delta_{\gamma_{0}} v_{q}$ is in $\bar{\alpha}_{k}$ if and only if $e_{q-1}^{\prime}$ is in $\bar{\alpha}_{k}$.

We repeat the process just described until we use all cycles of $\gamma_{0}, \gamma_{1}, \ldots, \gamma_{s}$. Since each edge of $1 \mathrm{k} v_{k}$ lies on some cycle, then we use all the edges of $1 \mathrm{k} v_{k}$ with the algorithm described above. At the end we will find that $\bar{\alpha}_{k}$ is written as a coboundary, i.e., $\bar{\alpha}_{k}=\sum_{v_{j k} \in \mathrm{lk} v_{k}} n_{j k} \delta_{\mathrm{lk} v_{k}} v_{j k} \in L_{0}$. From Lemma 4.4 it follows that $\alpha \in L_{0}$.

Let $\beta=\sum_{j=1}^{s} \sum_{\sigma_{i} \succ e_{j}} m_{i j}\left(\sigma_{i}, e_{j}\right) \in K_{1}$ and $\Gamma(\beta)$ be the graph spanned by all edges $e$ that appear in $\beta$. The next theorem gives a geometric interpretation of the 
chains in $K_{1}$ whose boundaries are in $L_{0}$; this result will be used in proving the main result for the general case, Theorem 5.6. Again we refer to Figure 1.

Theorem 4.6. Assume that the link of each vertex $v \in X$ is 2-connected, $\beta=$ $\sum_{j=1}^{s} \sum_{\sigma_{i} \succ e_{j}} m_{i j}\left(\sigma_{i}, e_{j}\right) \in K_{1}$ does not contain terms of $L_{1}$ (terms of the form $(\delta e, e))$, and $\partial^{\prime} \beta \in L_{0}$. Then the graph $\Gamma(\beta)$ is finite and has no vertices of valence 1 .

Proof. Suppose there is a vertex $v_{2}$ of valence 1 in $\Gamma(\beta)$. Let $e$ be the edge of $\Gamma(\beta)$ that has $v_{2}$ as a vertex, and let $v_{1}$ be the other vertex of $e$ (Figure 3). Let $\partial^{\prime} \beta_{v_{2}}:=\sum_{\sigma_{i} \succ v_{2}} n_{i 2}\left(\sigma_{i}, v_{2}\right)$, i.e., $\partial^{\prime} \beta_{v_{2}}$ is a sum only of those terms from $\partial^{\prime} \beta$ that have $v_{2}$ as a second coordinate. Define $\bar{\partial}^{\prime} \beta_{v_{2}}:=\sum_{\sigma_{i} \succ v_{2}} n_{i 2} e_{i 2}$, where $e_{i 2}$ is the face of $\sigma_{i}$ opposite $v_{2}\left(e_{i 2}\right.$ is an edge from lk $\left.v_{2}\right)$.

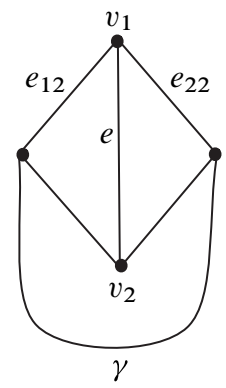

Figure 3

Since $e$ is an edge of $\Gamma(\beta)$, there exists at least one term with non-zero coefficient in $\beta$ whose second coordinate is $e$, and the first coordinate of that term is a 2-simplex that has $e$ as an edge. Hence at least one edge of $1 \mathrm{k} v_{2}$ that has $v_{1}$ as a vertex has a non-zero coefficient in $\bar{\partial}^{\prime} \beta_{v_{2}}$. Denote one of these edges by $e_{12}$. Since $\beta$ does not contain any terms of form $(\delta e, e)$, this means that at least one edge of lk $v_{2}$ which has $v_{1}$ as a vertex is not present in $\bar{\partial}^{\prime} \beta_{v_{2}}$, i.e., has a coefficient 0 in $\bar{\partial}^{\prime} \beta_{v_{2}}$. Denote by $e_{22}$ one of these edges. By Lemma 4.3, $e_{12}$ and $e_{22}$ lie on a cycle $\gamma$. Since the valence of $v_{2}$ in $\Gamma(\beta)$ is 1 , all edges on $\gamma$ other than $e_{12}$ and $e_{22}$ have coefficient 0 in $\bar{\partial}^{\prime} \beta_{v_{2}}$, $e_{22}$ has a coefficient 0 as well, and $e_{12}$ has a coefficient 1 . Therefore, the sum of the coefficients on $\gamma$ is 1 , which is not possible since $\partial^{\prime} \beta \in L_{0}$ and, by Theorem 4.5 , the sum of the coefficients on $\gamma$ must be 0 . Hence there exists at least one more edge in $\Gamma(\beta)$ that has $v_{2}$ as vertex different from $e$, i.e., the valence of $v_{2}$ is at least two.

\section{The Main Theorem}

The following two examples suggest that we need further hypotheses on $X$. 
Example 5.1. Let $X$ be the simplicial complex obtained by triangulating $\{(x, y, z) \in$ $\left.\mathbb{R}^{3} \mid x^{2}+y^{2}=1\right\} \cup\left\{(x, y, z) \mid x^{2}+y^{2} \leq 1, z=0\right\}$. This complex $X$ is CohenMacaulay. The link of each vertex is either homeomorphic to either $S^{1}$ or the letter $\theta$. Hence the link of each vertex is 2 -connected. But $H_{c}^{1}\left(X, \mathbb{Z}_{2}\right) \not 0$, i.e., $X$ is not one-ended.

Example 5.2. Let $X$ be a simplicial complex obtained by gluing two triangulated planes along a line. This simplicial complex is Cohen-Macaulay and the link of each vertex is 2 -connected. This complex is one-ended, i.e., $H_{c}^{1}\left(X, \mathbb{Z}_{2}\right) \cong 0$.

Recall that an edge $e$ of $X$ is singular if it is a face of more than two 2-simplices. By Lemma 4.1 the non-singular edges are those which are faces of exactly two 2simplices. One of the differences between these two examples is that the simplicial complex in Example 5.1 has a cycle all of whose edges are singular, and the complex in Example 5.2 does not.

We will introduce a new hypothesis on $X$. We will assume that $X$ is an infinite, 2-dimensional, acyclic, locally finite, path connected Cohen-Macaulay simplicial complex such that the link of each vertex is 2-connected and each cycle has a nonsingular edge.

Our goal is to show that $X$ has one end, i.e., $\operatorname{ker}\left\{H_{0}\left(L, \mathbb{Z}_{2}\right) \rightarrow H_{0}\left(K, \mathbb{Z}_{2}\right)\right\} \cong 0$, which by Theorem 3.3 , is equivalent to $H_{1}\left(X, \mathcal{L}_{\mathbb{Z}_{2}}^{2}\right) \cong 0$. Let $i_{*}: H_{0}\left(L ; \mathbb{Z}_{2}\right) \rightarrow$ $H_{0}\left(K ; \mathbb{Z}_{2}\right)$ be induced by the inclusion $i: L_{0} \hookrightarrow K_{0}$. Let $\left[\alpha_{0}\right] \in H_{0}\left(L ; \mathbb{Z}_{2}\right)$ and let $i_{*}\left(\left[\alpha_{0}\right]\right)=[0]$ (Figure 1). This means that $\alpha_{0}$ bounds in $K$, i.e., there exists a chain $\beta_{0} \in K_{1}$ such that $\partial^{\prime} \beta_{0}=\alpha_{0}$. We want to show that $\alpha_{0}$ bounds in $L$, i.e., $\left[\alpha_{0}\right]=[0]$. Therefore we need to show that there exists a chain $\beta \in L_{1}$ that is homologous to $\beta_{0}$ in $K$, i.e., $\partial^{\prime} \beta_{0}=\partial^{\prime} \beta=\alpha_{0}$.

Let $\beta_{0}=\sum_{j=1}^{s} \sum_{\sigma_{i} \succ e_{j}} m_{i j}\left(\sigma_{i}, e_{j}\right) \in K_{1}$. We may assume that $\beta_{0}$ is reduced with respect to $L_{1}$, i.e., $\beta_{0} \neq \beta_{0}^{\prime}+\beta_{0}^{\prime \prime}$ with $\beta_{0}^{\prime \prime} \in L_{1}$ and each term in $\beta_{0}^{\prime}$ appears in $\beta_{0}$. In other words, no subset of terms in $\beta_{0}$ add up to an element of $L_{1}$. Since $\beta_{0}$ does not have any terms of form $(\delta e, e)$, Theorem 4.6 implies that the graph $\Gamma\left(\beta_{0}\right)$ consisting of all edges that appear in $\beta_{0}$ does not have vertices of valence 1 .

Let $\gamma_{0}$ be a cycle in $\Gamma\left(\beta_{0}\right)$. There is a non-singular edge $e_{1}$ on $\gamma_{0}$. Let $\tau_{0}$ be the unique 2 -chain that bounds $\gamma_{0}$ ( $\gamma_{0}$ is a 1-cycle in the sense of the simplicial homology theory; since $X$ is 2-dimensional and acyclic, there is a unique 2-chain $\tau_{0}$ that bounds $\left.\gamma_{0}\right)$. We will denote the support of $\tau_{0}$ by $C\left(\gamma_{0}\right)$. Let $e_{1}=\sigma_{1} \cap \sigma_{2}$, where $\sigma_{1}$ and $\sigma_{2}$ are 2-simplices of $X, \sigma_{1} \in C\left(\gamma_{0}\right)$, and $\sigma_{2} \notin C\left(\gamma_{0}\right)$.

Our goal is to show that $\beta_{0}$ is homologous to an element of $L_{1}$. Let

$$
\beta_{0}^{\prime}:=\beta_{0}+\partial\left(\sigma_{1}, \sigma_{1}\right)=\beta_{0}+\left(\sigma_{1}, e_{1}\right)+\left(\sigma_{1}, e_{2}\right)+\left(\sigma_{1}, e_{3}\right)
$$

where $e_{1}, e_{2}$, and $e_{3}$ are edges of $\sigma_{1}$.

Let

$$
\beta_{1}:=\beta_{0}^{\prime}+\left(\text { terms of } \beta_{0}^{\prime} \text { that are in } L_{1}\right)
$$


Since we work with $\mathbb{Z}_{2}$ coefficients, $\beta_{1}$ does not have any terms of $L_{1}$ and $\partial \beta_{1} \in L_{0}$. By Theorem 4.6, the graph $\Gamma\left(\beta_{1}\right)$ does not have any vertices of valence 1 .

Proposition 5.4. The edge $e_{1}$ is not an edge of $\Gamma\left(\beta_{1}\right)$.

Proof. Since $e_{1}$ is a non-singular edge of the graph $\Gamma\left(\beta_{0}\right)$ it follows that one of the terms $\left(\sigma_{1}, e_{1}\right)$ or $\left(\sigma_{2}, e_{1}\right)$ is in $\beta_{0}$, but not both.

Case 1 . Let $\left(\sigma_{1}, e_{1}\right)$ be in $\beta_{0}$. Then

$\beta_{0}^{\prime}=\beta_{0}+\partial\left(\sigma_{1}, \sigma_{1}\right)=\left(\beta_{0}+\left(\sigma_{1}, e_{1}\right)\right)+\left(\sigma_{1}, e_{1}\right)+\left(\sigma_{1}, e_{1}\right)+\left(\sigma_{1}, e_{2}\right)+\left(\sigma_{1}, e_{3}\right)$

so

$$
\beta_{0}^{\prime}=\left(\beta_{0}+\left(\sigma_{1}, e_{1}\right)\right)+\left(\sigma_{1}, e_{2}\right)+\left(\sigma_{1}, e_{3}\right) .
$$

Since $\left(\sigma_{1}, e_{1}\right)$ is in $\beta_{0}$, the term $\left(\sigma_{1}, e_{1}\right)$ is not in $\left(\beta_{0}+\left(\sigma_{1}, e_{1}\right)\right)$. Since $\beta_{0}$ does not contain $\left(\sigma_{2}, e_{1}\right)$, it follows that $\beta_{0}^{\prime}$ does not have any terms with second coordinate $e_{1}$. Thus $\beta_{1}$ does not have a term with a second coordinate $e_{1}$. Hence $e_{1}$ is not an edge of $\Gamma\left(\beta_{1}\right)$.

Case 2. Let $\left(\sigma_{2}, e_{1}\right)$ be in $\beta_{0}$. Then

$\beta_{0}^{\prime}=\beta_{0}+\partial\left(\sigma_{1}, \sigma_{1}\right)=\left(\beta_{0}+\left(\sigma_{2}, e_{1}\right)\right)+\left(\left(\sigma_{2}, e_{1}\right)+\left(\sigma_{1}, e_{1}\right)\right)+\left(\sigma_{1}, e_{2}\right)+\left(\sigma_{1}, e_{3}\right)$

so

$$
\beta_{0}^{\prime}=\left(\beta_{0}+\left(\sigma_{2}, e_{1}\right)\right)+\left(\delta e_{1}, e_{1}\right)+\left(\sigma_{1}, e_{2}\right)+\left(\sigma_{1}, e_{3}\right) .
$$

Since $\left(\sigma_{2}, e_{1}\right)$ is in $\beta_{0}$, the term $\left(\sigma_{2}, e_{1}\right)$ is not in $\left(\beta_{0}+\left(\sigma_{2}, e_{1}\right)\right)$. The only term with a second coordinate $e_{1}$ in $\beta_{0}^{\prime}$ is $\left(\delta e_{1}, e_{1}\right)$, and this term is not in $\beta_{1}$ since it is in $L_{1}$. Therefore $\beta_{1}$ does not contain any terms with a second coordinate $e_{1}$, so $e_{1}$ is not an edge of $\Gamma\left(\beta_{1}\right)$.

We say that $X$ is strongly 1-acyclic if for any finite subcomplex $Y$ of $X$ there exists a finite subcomplex $F$ such that $Y \subseteq F$ and $H_{1}\left(F ; \mathbb{Z}_{2}\right) \cong 0$.

Proposition 5.5. Let $X$ be an acyclic 2-dimensional simplicial complex that is strongly 1-acyclic. Then for each finite graph $\Gamma$ in $X$ there exists a unique minimal subcomplex $C(\Gamma)$ such that $\Gamma \subset C(\Gamma)$ and $H_{1}\left(C(\Gamma) ; \mathbb{Z}_{2}\right) \cong 0$.

Proof. Since $X$ is strongly 1 -acyclic, there exists a subcomplex $F$ such that $\Gamma \subset F$ and $H_{1}\left(F ; \mathbb{Z}_{2}\right) \cong 0$. Let $C(\Gamma):=\bigcap_{\alpha} F_{\alpha}$ where the intersection is over all such subcomplexes $F_{\alpha}$ that contain $\Gamma$. It is clear that $\Gamma \subset C(\Gamma)$. We need to show that $H_{1}\left(C(\Gamma) ; \mathbb{Z}_{2}\right) \cong 0$. Let $z$ be a 1 -cycle in $C(\Gamma)$. Then $z$ is a 1 -cycle in $F_{\alpha}$ for each $\alpha \in A$. Assume that $z$ bounds a 2-chain $\tau_{\alpha_{1}}$ in $F_{\alpha_{1}}$ and a 2-chain $\tau_{\alpha_{2}}$ in $F_{\alpha_{2}}$. If $\tau_{\alpha_{1}} \neq \tau_{\alpha_{2}}$, then $\tau_{\alpha_{1}}+\tau_{\alpha_{2}}$ forms a non-zero 2-cycle in $X$ which is not possible since $X$ is acyclic and 2-dimensional. Hence $\tau_{\alpha_{1}}=\tau_{\alpha_{2}}$. 
If $X$ is strongly 1 -acyclic, we say an edge $e$ of a finite subgraph $\Gamma$ of $X$ is free if only one of the 2-simplices that have $e$ as an edge is in $C(\Gamma)$.

Now we are ready for the main result in the general case when $X$ is an infinite complex. Again, we refer to Figure 1.

Theorem 5.6. Let $X$ be an infinite, 2-dimensional, locally finite, acyclic, CohenMacaulay simplicial complex with the following properties:

(i) The link of each vertex of $X$ is 2-connected.

(ii) Any cycle in $X$ has a non-singular edge.

(iii) $X$ is strongly 1-acyclic.

If for all $\beta \in K_{1}$ such that $\partial^{\prime} \beta \in L_{0}, \beta$ is reduced with respect to $L_{1}$ and $\Gamma(\beta)$ has a non-singular free edge, then $H_{1}\left(X, \mathcal{L}_{\mathbb{Z}_{2}}^{2}\right) \cong 0$ (equivalently, $X$ has one end).

Proof. Let $\beta_{0} \in K_{1}$ be such that $\partial^{\prime} \beta_{0} \in L_{0}$ and $\beta_{0}$ is reduced with respect to $L_{1}$. Let $e_{1}$ be a non-singular free edge of $\Gamma\left(\beta_{0}\right)$, let $\sigma_{1}$ and $\sigma_{2}$ be the 2-simplices of $X$ that have $e_{1}$ as an edge with $\sigma_{1} \in C\left(\Gamma\left(\beta_{0}\right)\right)$ and $\sigma_{2} \notin C\left(\Gamma\left(\beta_{0}\right)\right)$. Let $e_{2}$ and $e_{3}$ be the other two edges of $\sigma_{1}$. We will define $\beta_{1}$ in the same way as we did in equation (5.3). We will show that $\left|C\left(\Gamma\left(\beta_{1}\right)\right)\right|_{2}<\left|C\left(\Gamma\left(\beta_{0}\right)\right)\right|_{2}\left(|*|_{2}\right.$ denotes the number of 2-simplices in $*$ ).

From Proposition 5.4 it follows that $e_{1}$ is not in $\Gamma\left(\beta_{1}\right)$. Since $\sigma_{1} \in C\left(\Gamma\left(\beta_{0}\right)\right)$, it follows that $e_{2}, e_{3} \in C\left(\Gamma\left(\beta_{0}\right)\right)$. Hence, $C\left(\Gamma\left(\beta_{0}\right)\right)$ contains $\Gamma\left(\beta_{1}\right)$. By the minimality of $C\left(\Gamma\left(\beta_{1}\right)\right)$ it follows that $C\left(\Gamma\left(\beta_{1}\right)\right) \subseteq C\left(\Gamma\left(\beta_{0}\right)\right)$.

We will show that $\sigma_{1} \notin C\left(\Gamma\left(\beta_{1}\right)\right)$. Since $e_{1}$ is a free edge of $C\left(\Gamma\left(\beta_{0}\right)\right)$, i.e., $\sigma_{1} \in$ $C\left(\Gamma\left(\beta_{0}\right)\right)$ and $\sigma_{2} \notin C\left(\Gamma\left(\beta_{0}\right)\right)$, by an elementary collapse operation on $\sigma_{1}, C\left(\Gamma\left(\beta_{0}\right)\right)$ deformation retracts to a subcomplex $C\left(\Gamma\left(\beta_{0}\right)\right)^{\prime}$. Hence $C\left(\Gamma\left(\beta_{0}\right)\right)^{\prime} \subset C\left(\Gamma\left(\beta_{0}\right)\right)$, $\sigma_{1} \notin C\left(\Gamma\left(\beta_{0}\right)\right)^{\prime}$, and $\left|C\left(\Gamma\left(\beta_{0}\right)\right)^{\prime}\right|_{2}<\left|C\left(\Gamma\left(\beta_{0}\right)\right)\right|_{2}$. Since $C\left(\Gamma\left(\beta_{0}\right)\right)$ is $\mathbb{Z}_{2}$-acyclic, it follows that $C\left(\Gamma\left(\beta_{0}\right)\right)^{\prime}$ is $\mathbb{Z}_{2}$-acyclic too. Since $e_{1}$ is not an edge of $\Gamma\left(\beta_{1}\right), e_{2}, e_{3} \in$ $C\left(\Gamma\left(\beta_{0}\right)\right)^{\prime}$ and all other edges of $\Gamma\left(\beta_{1}\right)$ are in $C\left(\Gamma\left(\beta_{0}\right)\right)^{\prime}$ (they are edges of $\Gamma\left(\beta_{0}\right)$, so they are in $\left.C\left(\Gamma\left(\beta_{0}\right)\right)^{\prime}\right)$, it follows that $\Gamma\left(\beta_{1}\right)$ is contained in $C\left(\Gamma\left(\beta_{0}\right)\right)^{\prime}$. By the minimality of $C\left(\Gamma\left(\beta_{1}\right)\right)$, and the fact that $H_{1}\left(C\left(\Gamma\left(\beta_{0}\right)\right)^{\prime} ; \mathbb{Z}_{2}\right) \cong 0$, it follows that $C\left(\Gamma\left(\beta_{1}\right)\right) \subseteq C\left(\Gamma\left(\beta_{0}\right)\right)^{\prime}$, and $\left|C\left(\Gamma\left(\beta_{1}\right)\right)\right|_{2} \leq\left|C\left(\Gamma\left(\beta_{0}\right)\right)^{\prime}\right|_{2}<\left|C\left(\Gamma\left(\beta_{0}\right)\right)\right|_{2}$. Now we have

$$
\beta_{0}-\beta_{1}=\partial^{\prime} c_{1}+l_{1}
$$

where $c_{1} \in K_{2}, l_{1} \in L_{1}$ and $\left|C\left(\Gamma\left(\beta_{1}\right)\right)\right|_{2}<\left|C\left(\Gamma\left(\beta_{0}\right)\right)\right|_{2}$.

We proceed by induction to find $\beta_{2}, \beta_{3}$, etc.

After the $i$ th step we have

$$
\beta_{i-1}-\beta_{i}=\partial^{\prime} c_{i}+l_{i}
$$

where $c_{i} \in K_{2}, l_{i} \in L_{1}$ and $\left|C\left(\Gamma\left(\beta_{i}\right)\right)\right|_{2}<\left|C\left(\Gamma\left(\beta_{i-1}\right)\right)\right|_{2}$. Since $C\left(\Gamma\left(\beta_{0}\right)\right)$ is finite, after some $k$ th step we will get $\beta_{k}$ such that $\left|C\left(\Gamma\left(\beta_{k}\right)\right)\right|_{2}=0$. Hence $\Gamma\left(\beta_{k}\right)$ is the empty graph, or every component of $\Gamma\left(\beta_{k}\right)$ is a finite tree; the latter is not possible 
because $\Gamma\left(\beta_{k}\right)$ does not have vertices of valence 1 . Hence $\Gamma\left(\beta_{k}\right)$ is the empty graph and $\beta_{k}=0$.

If we add the equations (5.7) for $i=1,2, \ldots, k$ we will get

$$
\beta_{0}-\beta_{k}=\sum_{i=1}^{k} \partial^{\prime}\left(c_{i}\right)+\sum_{i=1}^{k} l_{i} .
$$

Let $\beta=\sum_{i=1}^{k} l_{i}$. Then $\beta \in L_{1}$. Since $\beta_{k}=0$ it follows that

$$
\beta_{0}-\beta=\sum_{i=1}^{k} \partial^{\prime}\left(c_{i}\right) .
$$

Let $i_{*}: H_{0}\left(L ; \mathbb{Z}_{2}\right) \rightarrow H_{0}\left(K ; \mathbb{Z}_{2}\right)$ be induced by the inclusion $i: L_{0} \hookrightarrow K_{0}$. Let $\left[\alpha_{o}\right] \in H_{0}\left(L ; \mathbb{Z}_{2}\right)$ and let $i_{*}\left(\left[\alpha_{0}\right]\right)=[0]$. This means that $\alpha_{0}$ bounds in $K$, i.e., there exists a chain $\beta_{0} \in K_{1}$ such that $\partial^{\prime} \beta_{0}=\alpha_{0}$.

But we have shown that there exists a chain $\beta \in L_{1}$ that is homologous to $\beta_{0}$ in $K$, i.e., $\partial^{\prime} \beta_{0}=\partial^{\prime} \beta^{\prime}=\alpha_{0}$. Hence $\alpha_{0}$ bounds in $L$, i.e., $\left[\alpha_{0}\right]=[0]$. Therefore ker $i_{*}=0$, and, by Lemma 3.3, it follows that $H_{1}\left(X, \mathcal{L}_{\mathbb{Z}_{2}}^{2}\right) \cong 0$.

\section{Application of the Main Theorem}

We now apply Theorem 5.6 to the case where $X$ is the universal cover of a finite, 2-dimensional, aspherical, Cohen-Macaulay simplicial complex such that the link of each vertex is 2 -connected. We will denote this finite complex by $\Delta$. We wish to understand when $\widetilde{\Delta}$ has one end. We assume that $\widetilde{\Delta}$ is a simplicial complex and that $p: \widetilde{\Delta} \rightarrow \Delta$, the universal covering projection, is simplicial. We will abuse notation by using the same notation for an abstract simplicial complex and for the associated polyhedron.

Let $S$ be the subgraph of $\widetilde{\Delta}$ generated by all singular edges. If $S=\emptyset$, then $\widetilde{\Delta}$ is homeomorphic to $\mathbb{R}^{2}$ ( $\Delta$ is Cohen-Macaulay, and the links of all vertices are connected). Hence, $\widetilde{\Delta}$ is one-ended. Therefore the case $S=\emptyset$ is trivial. From now on we will assume that $S \neq \emptyset$.

Lemma 6.1. If $S \neq \emptyset$, then the following are equivalent:

(i) For every vertex $v_{0} \in p(S), i_{\#}: \pi_{1}\left(p(S), v_{0}\right) \rightarrow \pi_{1}\left(\Delta, v_{0}\right)$ is injective and $\pi_{1}\left(p(S), v_{0}\right)$ is non-trivial.

(ii) Each component of $S$ is a non-compact tree.

Proof. (i) $\Longrightarrow$ (ii): Let $S_{\tilde{v}_{0}}$ be a the path component of $S$, with base point $\tilde{v}_{0}$ over $v$. We have $\pi_{1}\left(S_{\tilde{v}_{0}}, \tilde{v}_{0}\right) \cong \operatorname{ker} i_{\#}$ (by Theorem 3.4.9 [6]). Since $i_{\#}$ is an injection, $\pi_{1}\left(S_{\tilde{v}_{0}}, \tilde{v}_{0}\right) \cong\{1\}$, which implies that each component of $S$ is a tree. Since 
$\pi_{1}\left(p(S), v_{0}\right)$ is non-trivial and $p(S)$ is a graph, this must be a free group, hence infinite. Hence $S_{\tilde{v}_{0}}$ is non-compact.

(ii) $\Longrightarrow$ (i): Let $\tilde{v}_{0}$ be a vertex over $v_{0}$ and let $T$ be the component of $S$ that contains $\tilde{v}_{0}$. By (ii), $T$ is a non-compact tree. Since $p(T)$ is compact and $T$ is a non-compact tree, $\pi_{1}\left(p(T), v_{0}\right) \neq\{1\}$. Let $[\omega] \in \operatorname{ker} i_{\#}$, where $\omega$ is a loop at $v_{0}$ in $p(T)$ that bounds in $\Delta$. Then, $\omega$ lifts to a loop $\widetilde{\omega}$ in the tree $T$ (by Theorem 3.4.9 [6]) which therefore bounds in $T$. Hence, $\omega$ bounds in $p(T)$, and $\operatorname{ker} i_{\#} \cong\{1\}$. Therefore $i_{\#}$ is an injection.

For the rest of the paper PL stands for "piecewise linear" and a general reference is [10].

If $P=|K|$ is a polyhedron and $x \in P$, the link of $x$ in $P$, well defined up to PL homeomorphism, is $\mathrm{l}_{K^{\prime}} x$ where $K^{\prime}$ is a subdivision of $K$ having $x$ as a vertex. If the link of each point of $\widetilde{\Delta}-S$ is connected, then $\widetilde{\Delta}-S$ is an open 2-manifold.

Lemma 6.2. If the link of each point of $\widetilde{\Delta}-S$ is connected, where $S$ is the singular set of $\widetilde{\Delta}$, then $\widetilde{\Delta}-S$ is an open 2 -manifold.

Proof. Let $x \in \widetilde{\Delta}-S$. Let $\widetilde{\Delta}^{\prime}$ be a subdivision of $\widetilde{\Delta}$ having $x$ as a vertex. Then $\mathrm{k}_{\tilde{\Delta}^{\prime}} x$ is a finite graph in which the link of each vertex consists of two points. Thus $1 \mathrm{k}_{\tilde{\Delta}^{\prime}} x$ is a closed 1-manifold. Since the link of each point of $\widetilde{\Delta}-S$ is connected, it follows that $\mathrm{lk}_{\tilde{\Delta}^{\prime}} x$ is a circle. Hence $\widetilde{\Delta}-S$ is a 2-manifold without boundary.

If there were a component of $\widetilde{\Delta}-S$ that is compact, then that component would be closed and open in $\widetilde{\Delta}$ which is not possible since $\widetilde{\Delta}$ is connected and $S \neq \emptyset$. Therefore $\widetilde{\Delta}-S$ is an open 2-manifold.

If $L$ is a subcomplex of $\widetilde{\Delta}$, then the regular neighborhood $N(L)$ of $L$ in $\widetilde{\Delta}$ is the simplicial neighborhood of $\operatorname{sd} L$ in $\operatorname{sd} \widetilde{\Delta}$, i.e., $N(L)=\bigcup_{v \in \text { sd } L} \operatorname{st}_{\mathrm{sd}} \tilde{\Delta} v$, where $\operatorname{sd} L$ and sd $\widetilde{\Delta}$ denote the first barycentric subdivision of $L$ and $\widetilde{\Delta}$ respectively.

Let $M$ be a PL $n$-manifold. We say that a subpolyhedron $P \subset M$ is collared in $M$ if there exists a closed neighborhood $N$ of $P$ in $M$ such that $(N, P)$ is PL homeomorphic to $(P \times[0,1], P \times\{0\})$ and $P$ is bicollared in $M$ if there exists a closed neighborhood $N$ of $P$ in $M$ such that $(N, P)$ is PL homeomorphic to $(P \times[-1,1], P \times\{0\})$. We recall that if $M$ is a PL $n$-manifold with boundary, then $\partial M$ is always collared. The following lemma is a standard PL topology exercise and we will omit the proof.

Lemma 6.3. If $\widetilde{\Delta}-S$ is a PL 2-manifold and if $N$ is a regular neighborhood of $S$, then $V:=\operatorname{cl}_{\widetilde{\Delta}}(\widetilde{\Delta}-N)$ is a PL 2-manifold whose boundary $\partial V:=\operatorname{bd}_{\widetilde{\Delta}} N$ is a bicollared 1-manifold without boundary in $\widetilde{\Delta}$.

From now on we will write $N$ for a neighborhood of $S$ that has bicollared boundary, we write $\partial N$ for $\operatorname{bd}_{\widetilde{\Delta}} N$, and $V:=\operatorname{cl}_{\widetilde{\Delta}}(\widetilde{\Delta}-N)$. 
Lemma 6.4. Let $M$ be a component of $V$. Then

(i) $\partial M \neq \emptyset$;

(ii) if any component of $\partial M$ is a line then $M$ is non-compact;

(iii) if every component of $\partial M$ is a line then $M$ is contractible.

Proof. (i) Since $S \neq \emptyset$, by Lemma 6.3 any path from $S$ to int $\widetilde{\Delta}(M)$ meets $\partial N$, hence $\partial M$. Therefore $\partial M \neq \emptyset$.

(ii) is obvious.

(iii) We will show that $H_{1}(M ; \mathbb{Z}) \cong 0$. Since $\tilde{\Delta}$ is a simply connected, every 1 -cycle in $M$ bounds in $\widetilde{\Delta}$ and is therefore homologous to a 1-cycle in $\partial M$. Since every component of $\partial M$ is a line, the only one 1-cycle in $\partial M$ is 0 . Hence, every 1 -cycle in $M$ is homologous to 0 . By (i), $M$ has the homotopy type of a connected graph, so $\pi_{1}(M)$ is free. Since $H_{1}(M ; \mathbb{Z}) \cong 0$, and the rank of $\pi_{1}(M)$ is equal to the free abelian rank of $H_{1}(M ; \mathbb{Z})$, it follows that $\pi_{1}(M) \cong\{1\}$.

Since $\partial M \neq \emptyset, H_{2}(M ; \mathbb{Z}) \cong 0$, so by the Hurewicz Theorem, $\pi_{2}(M) \cong\{1\}$. Therefore $M$ is contractible.

By Lemma $6.3 \partial N$ is a 1-manifold without boundary. So each component of $\partial N$ is a line or a circle. In the view of Lemma 6.4 from now on we will also assume that every component of $\partial N$ is a line.

Corollary 6.5. Each component of $N_{0}:=p(N)$, of $V_{0}:=p(V)$, and of $\partial V_{0}:=$ $p(\partial V) \pi_{1}$-injects in $\Delta$.

In this situation the components $N_{0}(\alpha), V_{0}(\beta)$, and $\partial V_{0}(\alpha, \beta)$ of $N_{0}, V_{0}$, and $\partial V_{0}$ respectively are aspherical and each $\partial V_{0}(\alpha, \beta)$ meets one $N_{0}(\alpha)$ and one $V_{0}(\beta)$. Thus we have $G$ decomposed as the fundamental group of a finite graph of groups, where the vertex groups are $G_{\alpha}=\pi_{1}\left(N_{0}(\alpha)\right)$ and $H_{\beta}=\pi_{1}\left(V_{0}(\beta)\right)$ and the edge groups are $K_{\alpha \beta}=\pi_{1}\left(\partial V_{0}(\alpha, \beta)\right)$. Moreover, $\Delta$ is aspherical, i.e., $\widetilde{\Delta}$ is contractible. Thus $G$ acts on a Bass-Serre tree $T$, and by the usual construction we have a "structure map" $g: \widetilde{\Delta} \rightarrow T$ where $g^{-1}$ (each vertex) is a component of $N$ or of $V$ and $g^{-1}$ (each edge) is a (component of $\partial V) \times[0,1]$ glued at one end to a component of $N$ and at the other end to a component of $V$. This proves:

Lemma 6.6. Let $N(\alpha)$ be a component of $N$, and let $V(\beta)$ be a component of $V$ such that $\partial N(\alpha) \cap \partial V(\beta) \neq \emptyset$. Then $\partial N(\alpha)$ and $\partial V(\beta)$ have only one line in common.

Lemma 6.7. For any finite subcomplex $Y$ of $\widetilde{\Delta}$ there exists a contractible subcomplex $K$ of $\widetilde{\Delta}$ which contains $Y$.

Proof. Let $Y$ be a finite subcomplex of $\widetilde{\Delta}$. We will show that there exists a finite contractible subcomplex $K$ of $\widetilde{\Delta}$ containing $Y$. In this proof $N$ will denote a regular 
neighborhood of $S$. We may assume that $\widetilde{\Delta}$ is subdivided so that $N, V, Y \cap V$, and $Y \cap N$ are subcomplexes of $\tilde{\Delta}$.

The subcomplex $Y$ only has non-empty intersection with a finite number of components of $V$ and of $N$. Let $V\left(\beta_{1}\right)$ have non-empty intersection with $Y$. Since $V\left(\beta_{1}\right)$ has countably many boundary components and $Y$ is finite, it follows that $Y$ may have non-empty intersection only with a finite number of them, say with $\partial V\left(\alpha_{1}\right)^{\beta_{1}}$, $\partial V\left(\alpha_{2}\right)^{\beta_{1}}, \ldots, \partial V\left(\alpha_{k}\right)^{\beta_{1}}$ (it may happen that $Y$ does not intersect any of the boundary components of $\left.V\left(\beta_{1}\right)\right)$. Let $N\left(\alpha_{1}\right)^{\beta_{1}}, N\left(\alpha_{2}\right)^{\beta_{1}}, \ldots, N\left(\alpha_{k}\right)^{\beta_{1}}$ be the components of $N$ having $\partial V\left(\alpha_{1}\right)^{\beta_{1}}, \partial V\left(\alpha_{2}\right)^{\beta_{1}}, \ldots, \partial V\left(\alpha_{k}\right)^{\beta_{1}}$ as boundary components respectively. By Lemma 6.6, $\partial V\left(\beta_{1}\right)$ has only one line in common with each of $\partial N\left(\alpha_{i}\right)^{\beta_{1}}$. Let $Y\left(\beta_{1}\right)$ be the subcomplex of $Y$ that lies in $(\widetilde{\Delta}-S)\left(\beta_{1}\right) \cup S\left(\alpha_{1}\right)^{\beta_{1}} \cup S\left(\alpha_{2}\right)^{\beta_{1}} \cup \cdots \cup S\left(\alpha_{k}\right)^{\beta_{1}}$ where $(\widetilde{\Delta}-S)\left(\beta_{1}\right)$ is the component of $\widetilde{\Delta}-S$ that contains $V\left(\beta_{1}\right)$, and $S\left(\alpha_{i}\right)^{\beta_{1}}$ is the component of $S$ that is in $N\left(\alpha_{i}\right)^{\beta_{1}}$ for all $i=1,2, \ldots, k$.

Let $Y\left(\beta_{1}\right)^{\prime}:=Y\left(\beta_{1}\right) \cap V\left(\beta_{1}\right)$ be a subcomplex of $V\left(\beta_{1}\right)$. There exists a contractible subcomplex $K\left(\beta_{1}\right)^{\prime}$ of $V\left(\beta_{1}\right)$ that contains $Y\left(\beta_{1}\right)^{\prime}$, and $K\left(\beta_{1}\right)^{\prime} \cap \partial V\left(\alpha_{i}\right)^{\beta_{1}}$ is connected for all $i=1,2, \ldots, k$. Since $K\left(\beta_{1}\right)^{\prime} \cap \partial V\left(\alpha_{i}\right)^{\beta_{2}}$ is connected, it collapses in the regular neighborhood $N\left(\alpha_{1}\right)^{\beta_{1}}$ onto a connected subtree $T\left(\alpha_{i}\right)^{\beta_{1}}$ of $S\left(\alpha_{i}\right)^{\beta_{1}}$. Let $K\left(\alpha_{i}\right)^{\beta_{1}}$ be a contractible subcomplex of the regular neighborhood $N\left(\alpha_{i}\right)^{\beta_{1}}$ containing $Y \cap N\left(\alpha_{i}\right)^{\beta_{1}}$ such that $K\left(\alpha_{i}\right)^{\beta_{1}} \cap S\left(\alpha_{i}\right)^{\beta_{1}}$ is connected, and also $K\left(\alpha_{i}\right)^{\beta_{1}} \cap \partial V\left(\alpha_{i}\right)^{\beta_{1}}$ is connected.

Since each intersection $K\left(\beta_{1}\right)^{\prime} \cap K\left(\alpha_{i}\right)^{\beta_{1}}$ is contractible for all $i=1,2, \ldots, k$, $K\left(\beta_{1}\right):=K\left(\beta_{1}\right)^{\prime} \cup K\left(\alpha_{1}\right)^{\beta_{1}} \cup K\left(\alpha_{2}\right)^{\beta_{1}} \cup \cdots \cup K\left(\alpha_{k}\right)^{\beta_{1}}$ is contractible and contains $Y\left(\beta_{1}\right)$.

Next we will do the same with the subcomplex $Y\left(\beta_{2}\right)$ of $Y$ that lies in $(\widetilde{\Delta}-S)\left(\beta_{2}\right) \cup S\left(\alpha_{1}\right)^{\beta_{2}} \cup S\left(\alpha_{2}\right)^{\beta_{2}} \cup \cdots \cup S\left(\alpha_{l}\right)^{\beta_{2}}$ where $(\widetilde{\Delta}-S)\left(\beta_{2}\right)$ is the component of $\widetilde{\Delta}-S$ that contains $V\left(\beta_{2}\right)$, and $S\left(\alpha_{i}\right)^{\beta_{2}}$ is the component of $S$ that is in $N\left(\alpha_{i}\right)^{\beta_{2}}$ for all $i=1,2, \ldots, l$. As with the construction of $K\left(\beta_{1}\right)$, there exists a contractible subcomplex $K\left(\alpha_{2}\right)$ that contains $Y\left(\alpha_{2}\right)$.

There is at most one component of $N$ that has non-empty intersection with both $V\left(\beta_{1}\right)$ and $V\left(\beta_{2}\right)$ (this follows from Lemma 6.6). Suppose there exists a component $N(\alpha)$ such that $N(\alpha) \cap V\left(\beta_{1}\right) \neq \varnothing$ and $N(\alpha) \cap V\left(\beta_{1}\right) \neq \emptyset$, and let $S(\alpha)$ be the tree that has $N(\alpha)$ as a regular neighborhood. Then $K\left(\beta_{1}\right)$ and $K\left(\beta_{2}\right)$ are either disjoint or they intersect in a contractible subcomplex (their intersection is a connected subtree of $S(\alpha)$ ), so their union is contractible. If such $N(\alpha)$ does not exist then $K\left(\beta_{1}\right) \cup K\left(\beta_{2}\right)$ is a disjoint union of contractible subcomplexes. In either case, $K\left(\beta_{1}\right) \cup K\left(\beta_{2}\right)$ contains $Y\left(\beta_{1}\right) \cup Y\left(\beta_{2}\right)$.

We continue this way. The algorithm is finite because $Y$ is finite. At the end we will get either a contractible subcomplex $K$ that contains $Y$, or a disjoint union of contractible subcomplexes whose union contains $Y$. In the latter case, we will connect the components of $K$ by segments, to get a contractible subcomplex $K$ that contains $Y$.

Corollary 6.8. $\widetilde{\Delta}$ is strongly 1-acyclic. 
Lemma 6.9. Let $K$ be a finite 2-dimensional subcomplex of $\widetilde{\Delta}$. Then $K$ has a free non-singular edge, i.e., there exists a non-singular edge e such that only one of the 2-simplices of which e is an edge is in $K$.

Proof. Suppose that $K$ does not have any free edges. Let $\tau$ be the 2-chain that is a sum of all 2-simplices from $K$, i.e., $\tau=\sum_{\sigma \in K} \sigma$ (we work with $\mathbb{Z}_{2}$-coefficients). For each non-singular edge $e$ in $K$, both 2-simplices of which $e$ is an edge are in $\tau$ because $K$ does not have any free edges. Therefore, $\partial \tau$ does not contain any nonsingular edges, i.e., all edges of $\partial \tau$ are singular. Each component of the singular set $S$ is a tree. Since $\partial \tau$ is a 1-cycle, $\partial \tau$ is a sum of 1-cycles each supported in a component of $S \cap K$. But the only 1-cycle in a tree is the trivial one. Hence $\partial \tau=0$ which is a contradiction because $\widetilde{\Delta}$ is a 2-dimensional contractible simplicial complex and there are no non-trivial 2-cycles in $\widetilde{\Delta}$.

Our motivating question was: Let $\Delta$ be a finite, 2-dimensional, aspherical CohenMacaulay simplicial complex. Under what hypotheses does $\widetilde{\Delta}$ have one end?

The following lemma is true in a more general case, not necessarily a complex that is the universal cover of a finite complex.

Lemma 6.10. Let $X$ be an infinite, 2-dimensional, path connected, Cohen-Macaulay complex such that the link of each vertex is 2-connected and any cycle in $X$ has a non-singular edge. Then each component of $S$ is an (infinite) non-trivial tree without vertices of valence 1 in that tree.

Proof. Let $S^{\prime}$ be a component of $S$. Suppose that $v$ is a vertex of $S^{\prime}$ with valence 1 in $S^{\prime}$. This implies that $\mathrm{k} v$ is a graph with only one vertex, denoted by $w$, with valence greater than 2 . If we remove $w$ from the link together with the edges from lk $v$ that have $w$ as a vertex, we will disconnect lk $v$ which is not possible since each vertex of $X$ is 2-connected. Therefore $S^{\prime}$ does not have any vertices of valence 1 . Since every cycle of $X$ contains a non-singular edge, it follows that $S^{\prime}$ does not have any cycles. Hence $S^{\prime}$ is a tree.

Lemma 6.11. Let $\Delta$ be a finite, 2-dimensional, connected simplicial complex. Let $S_{0}$ be the singular set in $\Delta$ and assume that the link of each vertex of $\Delta-S_{0}$ is connected. If each component $C$ of $\partial N\left(S_{0}\right)$ (which is homeomorphic to a circle) $\pi_{1}$-injects into $\Delta$, then $i_{\#}: \pi_{1}\left(S_{0}, v_{0}\right) \rightarrow \pi_{1}\left(\Delta, v_{0}\right)$ is injective for every vertex $v_{0} \in S_{0}$.

Proof. Let $\omega: S^{1} \rightarrow S_{0}$ be a loop in $S_{0}$ such that $\omega$ is homotopically trivial in $\Delta$. Let $f: B^{2} \rightarrow \Delta$ be a map (by a "map" we understand a "continuous function") such that the restriction $f_{\mid S^{1}}=\omega$. Let $S_{0, \omega}$ be the component of $S_{0}$ that contains $\omega$. We think of $\left(B^{2}, S^{1}\right)$ as a PL pair. We may assume that $f$ is a PL map. Let $N\left(S_{0, \omega}\right)$ be a regular neighborhood of $S_{0, \omega}$, and let $C_{1}, C_{2}, \ldots, C_{k}$ be all the boundary components of $\partial N\left(S_{0, \omega}\right)\left(C_{1}, C_{2}, \ldots, C_{k}\right.$ are cycles $)$. Since $f$ maps the open 2-manifold $B^{2}-$ $f^{-1}\left(S_{0}\right)$ into the open 2-manifold $\Delta-S_{0}$, using transversality, we may make a small 
perturbation of $f$ to get a map $f_{1}: B^{2} \rightarrow \Delta$ such that $f_{1}^{-1}\left(C_{1} \cup C_{2} \cup \cdots \cup C_{k}\right)$ is a closed 1-manifold and $f_{\mid f^{-1}\left(S_{0}\right)}=f_{1_{\mid f-1}\left(S_{0}\right)}$. Note $f_{1}^{-1}\left(C_{1} \cup C_{2} \cup \cdots \cup C_{k}\right) \cap$ $f_{1}^{-1}\left(S_{0}\right)=\emptyset$.

Let $A \subset f_{1}^{-1}\left(C_{1} \cup C_{2} \cup \cdots \cup C_{k}\right)$ be a cycle. Let $R$ be the closed region of $\stackrel{\circ}{B}^{2}$ that lies inside $A$ ( $R$ is a disk by Schönflies Lemma). Assume $f_{1}(A) \subset C_{1}$. Since $C_{1}$ is $\pi_{1}$-injective in $\Delta$, we may redefine $f_{1}$ so that $f_{1}(R) \subset C_{1}$. Let $A_{1}$ be a cycle in $\stackrel{\circ}{B}^{2}$ that is disjoint from $A$ but very close to $A$, does not intersect any cycles from $f_{1}^{-1}\left(C_{1} \cup C_{2} \cup \cdots \cup C_{k}\right)$, and $A$ is in the closed region $R_{1}$ that is inside $A_{1}$. We want to show that, with small changes on $f_{1}$, we can get $f_{1}\left(A_{1}\right) \cap C_{1}=\emptyset$ (from now on we will use often "small" changes on $f_{1}$, but we will not use different notation; all "new" maps will be again denoted by $\left.f_{1}\right)$. $C_{1}$ is bicollared, hence $f_{1}\left(R_{1}\right)$ is on one side of $C_{1}$ in the bicollared neighborhood of $C_{1}$.

We will repeat the above on every cycle of $f_{1}^{-1}\left(C_{1} \cup C_{2} \cup \cdots \cup C_{k}\right)$. Then $f_{1}\left(B^{2}\right)$ is either in the regular neighborhood $N\left(S_{0, \omega}\right)$ or outside $N\left(S_{0, \omega}\right)$. Since $f_{1_{\mid S 1}}=\omega$, it follows that $f_{1}\left(B^{2}\right)$ is in the regular neighborhood $N\left(S_{0, \omega}\right)$. The regular neighborhood $N\left(S_{0, \omega}\right)$ strongly deformation retracts onto $S_{0, \omega}$, so by changing the map $f_{1}$ again, we can get that $f_{1}\left(B^{2}\right) \subset S_{0, \omega}$. Therefore $\omega$ is homotopically trivial in $S_{0, \omega}$, which implies that $S_{0, \omega} \pi_{1}$-injects into $\Delta$.

Theorem 6.12. Let $\Delta$ be a finite, 2-dimensional, aspherical, Cohen-Macaulay complex such that the link of each vertex of $\Delta$ is 2-connected and $\Delta$ does not consist of only one 2-simplex. Let $S_{0}$ be the singular set in $\Delta$. If each component $C$ of the boundary of a regular neighborhood of $S_{0} \pi_{1}$-injects into $\Delta$, then $\widetilde{\Delta}$ has one end. In particular, $\pi_{1}(\Delta)$ is 2-dimensional duality group.

Proof. The link of each point of $\widetilde{\Delta}-\widetilde{S}_{0}$ is connected because $\widetilde{\Delta}$ is Cohen-Macaulay.. By Lemma 6.11, it follows that $\pi_{1}\left(S_{0}, v_{0}\right)$ is non-trivial for every vertex $v_{0} \in S_{0}$, and $i_{\#}: \pi_{1}\left(S_{0}, v_{0}\right) \rightarrow \pi_{1}\left(\Delta, v_{0}\right)$ is injective. Hence $\pi_{1}\left(\Delta, v_{0}\right)$ is non-trivial, and $\widetilde{\Delta}$ is infinite.

By Lemma 6.1, each component of $\widetilde{S}_{0}$ is a non-compact tree. This is equivalent with the property that every cycle in $\widetilde{\Delta}$ has a non-singular edge. Since the link of each vertex of $\widetilde{\Delta}$ is 2-connected, it follows that every edge of $\widetilde{\Delta}$ is a face of at least two 2-simplices (by Corollary 4.2). By Corollary 6.8 it follows that $\widetilde{\Delta}$ is strongly 1-acyclic. Therefore, the conditions (ii) and (iii) of Theorem 5.6 are satisfied.

It remains to check the last hypothesis of Theorem 5.6. Let $\beta$ be a chain in $K_{1}$ that does not have any terms of $L_{1}$ and $\partial^{\prime} \beta \in L_{0}$ (Figure 1). We will show that the graph $\Gamma(\beta)(\Gamma(\beta)$ is the graph spanned by all edges that appear in $\beta)$ has a non-singular free edge, i.e., there exists a non-singular edge of $\Gamma(\beta)$ that is a face of two 2-simplices $\sigma_{1}$ and $\sigma_{2}$ such that $\sigma_{1} \in C(\Gamma(\beta))$ and $\sigma_{2} \notin C(\Gamma(\beta))$.

Suppose that $\Gamma(\beta)$ does not have a non-singular free edge. Since $\Gamma(\beta)$ does not have any vertex with valence 1 (by Theorem 4.6), it follows that $\Gamma(\beta)$ ) has at 
least one cycle. Hence $C(\Gamma(\beta))$ is 2-dimensional. By Lemma 6.9, $C(\Gamma(\beta))$ has a non-singular free edge $e$. By assumption, the edge $e$ is not in $\Gamma(\beta)$. Then, by an elementary collapse, we would get a subcomplex whose first homology is trivial, and contains $\Gamma(\beta)$, which contradicts the minimality of $C(\Gamma(\beta))$.

By Theorem $5.6, H_{1}\left(\widetilde{\Delta}, \mathcal{L}_{\mathbb{Z}_{2}}^{2}\right) \cong 0$, and by Theorem $2.4 H_{c}^{1}\left(\widetilde{\Delta} ; \mathbb{Z}_{2}\right) \cong 0$. Hence $\widetilde{\Delta}$ has one end.

Let $S_{1}, S_{2}, \ldots, S_{n_{1}}$ be the components of the singular set $S_{0}$ in $\Delta$ and $N_{i}$ ( $i=1,2, \ldots, n_{1}$ ) be the pairwise disjoint regular neighborhoods of $S_{1}, S_{2}, \ldots, S_{n_{1}}$ respectively. We write $N=\bigsqcup_{i=1}^{n_{1}} N_{i}$. The boundary of $N$ in $\Delta$ consists of circles $C_{j}\left(j=1,2, \ldots, n_{2}\right)$. The closure in $\Delta$ of each component of $\Delta-N$ is a compact surface whose boundary consists of some of the circles $C_{j}$ (each circle $C_{j}$ occurs exactly once as a boundary of one surface). We denote these surfaces by $M_{k}$ $\left(k=1,2, \ldots, n_{3}\right)$. Thus we express $\Delta$ as a graph of connected polyhedra; the vertex polyhedra are the $N_{i}$ s and the $M_{k} \mathrm{~s}$, the edge polyhedra are the $C_{j}$ s. Note that there is at least one $M_{k}$ because $\Delta$ has one free edge (Lemma 6.9) and $N$ collapses onto $S$. As a consequence of Theorem 6.12, we have the following corollary which provides necessary and sufficient conditions for $\widetilde{\Delta}$ to have one end.

Corollary 6.13. Let $\Delta$ be a finite, 2-dimensional, aspherical, Cohen-Macaulay complex such that the link of each vertex of $\Delta$ is 2-connected and $\Delta$ does not consist of only one 2-simplex. Assume that no surfaces $M_{k}$ is a disk. Then the following are equivalent:

(i) Each $C_{j} \pi_{1}$-injects into $N$.

(ii) $\widetilde{\Delta}$ has one end and semistable fundamental group at infinity.

Proof. It is obvious that this is true when $S_{0}=\emptyset$. Assume $S_{0} \neq \emptyset$.

We choose a maximal tree in $\Delta$, well behaved with respect to our graph-ofpolyhedra decomposition. ([6]). Assuming ( $i$ ), this gives a graph-of-groups decomposition of $\pi_{1}(\Delta)$ in which the vertex groups are (non-trivial) finitely generated free groups, and the edge groups are infinite cyclic (this is where we must rule out that any $M_{k}$ is a disk). By Britton's Lemma each $C_{j} \pi_{1}$-injects into $\Delta$. Thus Theorem 6.12 implies that $\widetilde{\Delta}$ has one end.

For the converse, we noted that there is at least one $C_{j}$. Assume some $C_{j}$ does not $\pi_{1}$-inject into $N$; thus the inclusion $C_{j} \hookrightarrow N$ induces the trivial homomorphism on $\pi_{1}$. Then we have a decomposition of $\pi_{1}(\Delta)$ as the fundamental group of graph of groups similar to the previous one except that some of the edge groups are trivial instead of being infinite cyclic. The set $S$ is non-empty, and Lemma 6.10 implies that each component of $N$ has non-trivial (free) fundamental group. Let $M$ be the surface having $C_{j}$ in its boundary. Then the decomposition includes an edge whose vertex groups are non-trivial while the edge groups are trivial. This is enough to ensure that $\pi_{1}(\Delta)$ has more than one end.

We have proved that (i) is equivalent to: 
(ii') $\pi_{1}(\Delta)$ is the fundamental group of a graph of groups where each vertex group is free (hence semistable at each end) and each edge group is infinite cyclic (hence finitely generated).

By the Main Theorem of [9], the properties in parentheses in (ii') are sufficient to imply that the group $\pi_{1}(\Delta)$ is semistable at infinity.

Remark. Perrin Wright, in [11], has shown that every finite, 2-dimensional polyhedron can be deformed to a finite fake surface. Triangulated fake surfaces are Cohen-Macaulay and the link of each vertex of a fake surface is 2-connected. Hence, if we consider a finite 2-dimensional, aspherical simplicial complex $K$, then $K$ can be deformed to a fake surface $\Delta$. According to Theorem $6.12, \widetilde{\Delta}$ has one end if each component of the boundary of a regular neighborhood of the singular set $S_{0}$ of $\Delta$ (which are circles) $\pi_{1}$-injects in $\Delta$. The question that we are considering is to identify the preimage of each of these circles in the simplicial complex $K$.

\section{A. Zeeman's spectral sequences}

In this section we give a proof of Theorem 2.4. The proof is based on the work of F. J.F. Lasheras [5] where he generalized Theorem 1 from [12] for locally finite simplicial complexes (Theorem 1 in [12] is for finite simplicial complexes). In [12], Zeeman generalized the Poincaré duality for manifolds. The exposition in this section is based on [5], [8], and [7].

Let $X$ be an $n$-dimensional, locally finite simplicial complex. The coefficients are in a commutative ring $R$ with $1 \neq 0$ and will be omitted. We define $B^{r, s}$ to be a bigraded module in the following way: If $0 \leq s \leq r \leq n$ we define $B^{r, s}$ to be the free abelian group generated by all pairs of simplices $(\tau, \sigma)$ such that $\operatorname{dim} \tau=r$, $\operatorname{dim} \sigma=s$, and $\tau \succ \sigma$; we define $B^{r, s}:=0$ otherwise. We have:

$$
\begin{gathered}
d_{1}: B^{r, s} \rightarrow B^{r+1, s}, \quad d_{1}((\tau, \sigma)):=(\delta \tau, \sigma), \\
d_{2}: B^{r, s} \rightarrow B^{r, s-1}, \quad d_{2}((\tau, \sigma)):=(-1)^{r}(\tau, \partial \sigma), \\
d_{1} \circ d_{1}=0=d_{2} \circ d_{2}, \\
d_{1} \circ d_{2}+d_{2} \circ d_{1}=0 .
\end{gathered}
$$

Then $\left\{B^{r, s} ; d_{1}, d_{2}\right\}$ is a double complex. We associate to this double complex a chain complex $\left\{B_{*}, d\right\}$ defined as follows:

$$
d:=d_{1}+d_{2}: B_{m} \rightarrow B_{m-1}, \quad d((\tau, \sigma)):=(\delta \tau, \sigma)+(-1)^{r}(\tau, \partial \sigma)
$$

where $B_{m}:=\bigoplus_{s-r=m} B^{r, s}$.

There are two spectral sequences ${ }_{\mathrm{I}} E^{*}$ and ${ }_{\mathrm{II}} E^{*}$ such that

$$
\begin{array}{cl}
{ }_{\mathrm{I}} E_{p, q}^{2}=H^{p}\left(H_{q}\left(B ; d_{2}\right) ; \bar{d}_{1}\right), & 0 \leq q \leq p \leq n, \\
{ }_{\mathrm{II}} E_{p, q}^{2}=H_{p}\left(H^{q}\left(B ; d_{1}\right) ; \bar{d}_{2}\right), & 0 \leq p \leq q \leq n,
\end{array}
$$


and the first spectral sequence converges to $H_{q-p}(B ; d)$, and the second one converges to $H_{p-q}(B ; d)$. The differentials $\bar{d}_{1}$ and $\bar{d}_{2}$ are induced by $d_{1}$ and $d_{2}$ respectively. (Theorem 2.15, [8])

Lemma A.1. The spectral sequence ${ }_{\mathrm{I}} E$ collapses to an isomorphism

$$
H_{m}(B) \cong H_{c}^{-m}(X) \text {. }
$$

Proof. Let $C^{t}:=H_{q}\left(B^{t, *} ; d_{2}\right)$. Then

$$
C^{t}=H_{q}\left(\bigoplus_{\operatorname{dim} \tau=t} B^{t, *} ; d_{2}\right) \cong \bigoplus_{\operatorname{dim} \tau=t} H_{q}\left(B_{*}^{\tau} ; d_{2}\right)
$$

The free abelian group $B_{*}^{\tau}$ is generated by all pairs $(\tau, \sigma)$ such that $\operatorname{dim} \tau=t$, $\operatorname{dim} \sigma=*$, and $\tau \succ \sigma$. Hence $H_{q}\left(B_{*}^{\tau} ; d_{2}\right) \cong H_{q}(C(\tau) ; \partial)$ where $C(\tau)$ is the simplicial chain complex for $\tau$. Then $C^{t} \cong \bigoplus_{\underline{d i m} \tau=t} R$ when $q=0$, and $C^{t} \cong 0$ when $q \neq 0$. Hence ${ }_{\mathrm{I}} E_{p, 0}^{2}=H^{p}\left(H_{q}\left(B ; d_{2}\right) ; \bar{d}_{1}\right) \cong H^{p}\left(\left\{C^{t}\right\}_{t=0}^{n} ; \delta\right) \cong H_{c}^{p}(X)$, and ${ }_{\mathrm{I}} E_{p, q}^{2} \cong 0$ when $q \neq 0$. On the other hand

$$
0 \cong{ }_{\mathrm{I}} E_{-p+2,-1}^{2} \stackrel{d^{2}}{\longrightarrow}{ }_{\mathrm{I}} E_{-p, 0}^{2} \stackrel{d^{2}}{\longrightarrow}{ }_{\mathrm{I}} E_{-p-2,1}^{2} \cong 0 .
$$

Therefore ${ }_{\mathrm{I}} E_{-p, 0}^{\infty} \cong{ }_{\mathrm{I}} E_{-p, 0}^{2}$. Since ${ }_{\mathrm{I}} E_{p, q}^{2} \cong 0$ when $q \neq 0$, it follows that ${ }_{\mathrm{I}} E_{p, q}^{\infty} \cong$ ${ }_{\mathrm{I}} E_{p, q}^{2} \cong 0$ when $q \neq 0$. Since ${ }_{\mathrm{I}} E_{p, q}^{2}$ converges to $H_{q-p}(B ; d)$, it follows that ${ }_{\mathrm{I}} E_{-p, 0}^{2} \cong{ }_{\mathrm{I}} E_{-p, 0}^{\infty} \cong H_{p}(B ; d)$. Therefore $H_{p}(B ; d) \cong H_{c}^{-p}(X)$.

Lemma A.2. The spectral sequence ${ }_{\mathrm{II}} E$ runs:

$$
{ }_{\text {II }} E_{p, q}^{2} \cong H_{p}\left(X ; \mathcal{L}_{X}^{q}\right) \Longrightarrow H_{c}^{q-p}(X) .
$$

Proof. ${ }_{\mathrm{II}} E_{p, q}^{2}=H_{p}\left(H^{q}\left(B ; d_{1}\right) ; \bar{d}_{2}\right)$ converges to $H_{p-q}(B ; d)$ which is isomorphic to $H_{c}^{q-p}(X)$ by the previous lemma.

Let

$$
C_{t}:=H^{q}\left(B^{*, t} ; d_{1}\right)=H^{q}\left(\bigoplus_{\operatorname{dim} \sigma=t} B_{\sigma}^{*} ; d_{1}\right) \cong \bigoplus_{\operatorname{dim} \sigma=t} H^{q}\left(B_{\sigma}^{*} ; d_{1}\right)
$$

where $B_{\sigma}^{*}$ is the free abelian group generated all pairs $(\tau, \sigma)$, where $\operatorname{dim} \tau=*$, $\operatorname{dim} \sigma=t$, and $\tau \succ \sigma$. Hence $H^{q}\left(B_{\sigma}^{*} ; d_{1}\right) \cong H^{q}(X, X-\stackrel{\text { st }}{\sigma} ; \delta)$. Next

$$
{ }_{\mathrm{II}} E_{p, q}^{2}=H_{p}\left(\left\{\bigoplus_{\operatorname{dim} \sigma=t} H^{q}(X, X-\stackrel{\circ}{\mathrm{st}} \sigma)\right\}_{t=0}^{n} ; d_{2}\right) \cong H_{p}\left(X ; \mathcal{L}_{X}^{q}\right) .
$$

Hence $_{\mathrm{II}} E_{p, q}^{2} \cong H_{p}\left(X ; \mathcal{L}_{X}^{q}\right) \Longrightarrow H_{c}^{q-p}(X)$.

A path connected, finite dimensional, locally finite simplicial complex $\Delta$ is CohenMacaulay if for all $\sigma \in \Delta, \widetilde{H}_{i}(\mathrm{lk} \sigma) \cong 0$ for all $i<\operatorname{dim}(\operatorname{lk} \sigma)$. It follows that if $X$ is Cohen-Macaulay complex, then for any simplex $\sigma \in X$ we have $\tilde{H}^{i}(\mathrm{lk} \sigma) \cong 0$ for all $i<\operatorname{dim}(\operatorname{lk} \sigma)$. 
Next we will prove Theorem 2.4.

Theorem 2.4. Let $X$ be an $n$-dimensional, locally finite, Cohen-Macaulay simplicial complex. Then for all $p \in \mathbb{Z}$

$$
H_{p}\left(X, \mathcal{L}_{X}^{n}\right) \cong H_{c}^{n-p}(X)
$$

Proof. Since $X$ is Cohen-Macaulay, it follows that

$$
H^{q}(X, X-\stackrel{\circ}{\mathrm{st}} \sigma) \cong \tilde{H}^{q-\operatorname{dim} \sigma-1}(\mathrm{lk} \sigma) \cong 0
$$

when $q \neq n$. Hence $_{\mathrm{II}} E_{p, q}^{2} \cong 0$ when $q \neq n$, and ${ }_{\mathrm{II}} E_{p, q}^{\infty} \cong{ }_{\mathrm{II}} E_{p, q}^{2} \cong 0$ when $q \neq n$. Then

$$
0 \cong{ }_{\mathrm{II}} E_{p+2, n-1}^{2} \stackrel{d^{2}}{\longrightarrow}{ }_{\mathrm{II}} E_{p, n}^{2} \stackrel{d^{2}}{\longrightarrow}{ }_{\mathrm{II}} E_{p-2, n+1}^{2} \cong 0 .
$$

Therefore $_{\mathrm{II}} E_{p, n}^{\infty} \cong{ }_{\mathrm{II}} E_{p, n}^{2}$, i.e., $H_{p}\left(X, \mathcal{L}_{X}^{n}\right) \cong{ }_{\mathrm{II}} E_{p, n}^{2} \cong H_{c}^{n-p}(X)$.

\section{References}

[1] R. Bieri, Homological dimension of discrete groups. 2nd ed., Queen Mary College Mathematical Notes, Queen Mary College, Department of Pure Mathematics, London 1981. Zbl 0357.20027 MR 715779

[2] N. Brady, J. McCammond, and J. Meier, Local-to-asymptotic topology for cocompact CAT(0) complexes. Topology Appl. 131 (2003), 177-188. Zbl 1045.20037 MR 1981872

[3] R. A.Brualdi, Introductory combinatorics. 5th ed., Pearson Prentice Hall, Upper Saddle River, NJ, 2010. MR 2655770

[4] W. Bruns and J. Herzog, Cohen-Macaulay rings. Cambridge Stud. Adv. Math. 39, Cambridge University Press, Cambridge 1993. Zbl 0788.13005 MR 1251956

[5] F. J. Fernandez-Lasheras, Fake Surfaces, Thickenings and cohomology of groups. Dissertation, SUNY Binghamton, Binghamton 1996.

[6] R. Geoghegan, Topological methods in group theory. Graduate Texts in Math. 243, Springer, New York 2008. Zbl 1141.57001 MR 2365352

[7] P. J. Hilton and U. Stammbach, A course in homological algebra. Graduate Texts in Math. 4, Springer-Verlag, New York-Berlin 1971. Zbl 0238.18006 MR 0346025

[8] J. McCleary, A user's guide to spectral sequences. 2nd ed., Cambridge Stud. Adv. Math. 58, Cambridge University Press, Cambridge 2001. Zbl 0959.55001 MR 1793722

[9] M. L. Mihalik and S. T. Tschantz, Semistability of amalgamated products and HNNextensions. Mem. Amer. Math. Soc. 98 (1992), no. 471. Zbl 0792.20027 MR 1110521

[10] C. P. Rourke and B. J. Sanderson, Introduction to piecewise-linear topology. Ergeb. Math. Grenzgeb. 69, Springer-Verlag, Berlin 1972. Zbl 0477.57003 MR 0350744

[11] P. Wright, Formal 3-deformations of 2-polyhedra. Proc. Amer. Math. Soc. 37 (1973), 305-308. Zbl 0253.57001 MR 0331397 
[12] E. C. Zeeman, Dihomology III. A generalization of the Poincaré duality for manifolds. Proc. London Math. Soc. (3) 13 (1963), 155-183. Zbl 0109.41302 MR 0153011

Received December 23, 2011; revised April 24, 2012

R. Atanasov, Department of Mathematics and Computer Science, Western Carolina University, Cullowhee, NC 28723, U.S.A.

E-mail: ratanasov@email.wcu.edu 